\title{
3rd Congress of the International Society of Nutrigenetics/Nutrigenomics (ISNN)
}

\section{Abstracts}

October 21-23, 2009,

Bethesda, Md., USA
Guest Editors

Artemis P. Simopoulos, Washington, D.C.

John Milner, Rockville, Md.

\section{Contents}

\section{Oral Presentations}

Session I: Frontiers in Nutrigenetics

Abstracts A1-A5

Session II: Frontiers in Nutrigenetics (continued)

Abstracts A6-A12

Session III: Frontiers in Epigenetics

Abstracts A13-A18

Session IV: The Impact of Transcriptomics on Nutrigenomics

Abstracts A19-A23

Session V: Noncoding RNAs and Posttranslational

Gene Regulation

Abstracts A24-A26

Session VI: Moving Beyond Genomics

Abstracts A27-A30

Session VII: Frontiers in Nutrigenetics/Nutrigenomics:

Building Partnerships

Abstracts A31-A36

Poster Presentations

Abstracts P1-P37 


\section{Journal of \\ Nutrigenetics \\ Nutrigenomics}

\section{Session I: Frontiers in Nutrigenetics}

\section{A1 \\ Opportunities and Challenges in Nutrigenetics/Nutrigenomics and Health}

\author{
Raffaele De Caterina \\ Gabriele d'Annunzio University School of Medicine, Chieti, \\ Italy
}

Like drugs, nutrients have the ability to interact with and modulate molecular mechanisms underlying an organism's physiological functions. Awareness of the different effects of nutrients according to our genetic constitution (nutrigenetics) and how nutrients may affect gene expression (nutrigenomics) is prompting a revolution in the field of nutrition. Nutritional sciences have always studied the effects of nutrients in terms of "average" responses, without much bothering about interindividual variability and the underlying causes. Performing population-scaled intervention nutritional (similarly to pharmacological) studies in the absence of genetic knowledge may result in erroneous scientific conclusions and misinformed nutritional recommendations, which may not suit the need of promoting health at the level of the single individual. The field of nutrition has now begun to take advantage of both technological advances and the supporting analytical software developed in the postgenomic area to more comprehensively probe the complex relationship between genes and diet. The creation of nutrigenomics and nutrigenetics, two fields with distinct approaches to elucidate the interaction between diet and genes but with the common ultimate goal of optimizing health through the personalization of diet, provides powerful approaches to unravel the complex relationships among nutritional molecules, genetic polymorphisms, and the biological system as a whole. Translated as the simple concept of "personalized nutrition," the promises of nutrigenetics/ nutrigenomics appear as a major step forward in the understanding of our responses to a major component-nutrition - of our changing environment. Thus, the concepts encompassed by the broad area of nutrigenetics/nutrigenomics, at the intersection of nutrition, genetics, and molecular and systems biology, constitute a major opportunity for advancing our knowledge to promote human health.

However, at least two major types of challenges lie ahead. One is the reluctance to embrace these new fields, primarily due to the fear of being unable to manage the overwhelming quantity of biological data that may be generated and that would need interpretation andto a large extent-simplification to be translated into a practical message. The danger of the consequent simplification would be to take the results of a single study on a very specific outcome, very often on intermediate (surrogate) endpoints, and to infer that such results are applicable to the complexity of a living organism, where no single organ or tissue is independent of the others. The second challenge is the need to be aware that the area of "personalized nutrition" is seen by many disguised amateurs as a golden opportunity for marketing enterprises before solid knowledge in any specific area is acquired. Although the first challenge is manageable by the ever-increasing availability of biomedical and statistical tools and the wisdom necessary in health inference - a general problem in medical science - the second challenge requires great attention and wisdom and poses altogether important ethical issues. A scientific society devoted to the study of nutrigenetics/nutrigenomics can indeed serve the commendable roles of (1) promoting science and favoring scientific communication and (2) permanently working as a "clearinghouse" to prevent disqualifying logical jumps, correct or stop unwarranted claims, and prevent the creation of unwarranted expectations in patients and in the general public.

\section{A2 \\ Role of Human Genetic Variation in Nutritional Epidemiology Research}

\section{David J. Hunter \\ Harvard School of Public Health, Boston, Mass., USA}

Objectives: To discuss the role that the recent surge in findings from genome-wide association studies (GWASs) may play in nutritional epidemiology research.

Methods: Review of GWASs was conducted for health endpoints such as cancer, as well as biomarkers of nutrient adequacy. The combination of the knowledge of human genetic variation obtained in the HapMap, with high-throughput methods to genotype DNA samples at hundreds of thousands of loci, has led to a major step forward in our ability to discover low-penetrance genetic variants related to a wide variety of phenotypes. With respect to the major cancers, more than 20 new loci have been found for prostate cancer and more than 15 for breast and colorectal cancers. By and large, these loci either have not been in genes previously suspected of being associated with these cancers or have not been in genes at all, leading to a new appreciation of the importance of intergenic regions in the heritability of these cancers. These new loci provide a new source of information on mechanisms of cancer, some of which may involve interaction with diet and metabolism.

In contrast, studies of nutrient biomarkers have "rediscovered" known variants, have established new variants in genes known to be involved in nutrient metabolism, and have discovered novel associations. GWASs of serum folate, for instance, have identified the MTHFR 677 variant, previously known to be an important determinant of blood levels. GWASs of vitamin B12 have identified variation in the cobalamin gene as important. However, the strongest association was with a gene not previously established as a major influence on vitamin B12 levels - the FUT2 gene-giving rise to new hypotheses about vitamin B12 absorption in humans.

\section{KARGER \\ Fax +41613061234}

E-Mail karger@karger.ch

www.karger.com
(C) 2010 S. Karger AG, Basel

Accessible online at: www.karger.com/jnn 
Another promising avenue in the intersection of genetic and nutritional research is the possibility that a form of inference known as Mendelian randomization may help us establish relationships between nutrient metabolism and disease. Although a nutrient biomarker may be associated with a disease outcome due to confounding (e.g., lifestyle factors) if a genetic predictor of the biomarker is associated with the outcome (and is less likely to be associated with lifestyle factors), then this may add to the evidence that the biomarker is causally related to the outcome.

Conclusions: The new knowledge of the relationships between inherited low-penetrance variants and nutrient intake and metabolism could have a profound impact on our ability to convincingly demonstrate nutritional influences on human health.

\section{A3 \\ Genome-Wide Association Studies and Diet}

Lynnette R. Ferguson

Discipline of Nutrition, Faculty of Medical and Health

Sciences, The University of Auckland, and Nutrigenomics

New Zealand, Auckland, New Zealand

The fields of nutrigenetics and nutrigenomics have a powerful potential to tailor diets to optimize health and wellness and reduce the risk of disease. It is known that many genetic variants, usually but not exclusively measured as single nucleotide polymorphisms (SNPs), affect the response of a cell to a nutrient. Many of the reported genediet interactions described to date depend on the identification of candidate genes, which have been identified through a priori knowledge of disease processes. However, genome-wide association studies (GWASs) are not only validating genes and SNPs hitherto anticipated by knowledge of biochemical pathways but also are revealing new gene-disease associations not anticipated from prior knowledge. Crohn's disease (CD) provides a good example. GWASs have confirmed the previously reported association of disease susceptibility with SNPs in various pattern-recognition receptors that affect host recognition of mucosal microbiota, as was predictable by prior knowledge. However, GWASs also have identified hitherto unexpected genes in this disease, including SNPs in the proinflammatory cytokine interleukin-23 receptor and autophagy-related 16-like 1. It is important that such methods are complemented with innovative methodologies for characterizing the likely impact of foods to take the field to another dimension of value for human diet development and optimized health.
A4

\section{Copy Number Variation and Human Dietary Adaptations From an Evolutionary Perspective}

Anne C. Stone

Arizona State University, Tempe, Ariz., USA

Objectives: Understanding our genome structure, variation, evolutionary history, and the adaptations that "make us human," including our dietary adaptations, requires comparisons with our closest relatives. Dietary adaptations can occur in multiple ways. Two studies investigated dietary adaptations in humans with comparisons to other primates. The first tests whether the phenylthiocarbamide (PTC) tasting variation found in both humans and chimpanzees is the result of the same genotypes at the TAS2R 38 gene in each species maintained via balancing selection. The second study examines whether copy number variation in the salivary amylase gene (AMY1) is linked to differences in starch digestion in the mouth and whether copy number is higher in populations that consume more starch. We also investigated the amylase genes in other primates to test whether these genes have changed over time and whether these changes correspond to changes in diet.

Methods: Buccal swabs were collected from 50 European Americans, 38 Hadza, and 17 Datog. Additional human DNA samples were obtained from the HGDP-CEPH human genome diversity cell line panel. Nonhuman primate DNA samples were extracted from blood samples or buccal swabs collected from zoos and primate centers. Experimental and analytical methods are described in Perry et al. (2007, Nature Genetics 39:1256-1260) and Wooding et al. (2006, Nature 440:930-934).

Results: Sequence analysis of the $T A S 2 R 38$ gene in humans and chimpanzees reveals that the tasting and nontasting alleles are different in each species. The tasting and nontasting phenotypes inferred from the sequence results were confirmed through observations of chimpanzees given fruit soaked in PTC or water. The comparison of primate amylase genes revealed that only humans have copy number variation at the $A M Y 1$ gene and that this gene appears to be a pseudogene in bonobos. Saliva from European Americans was tested, and a positive correlation was found between higher copy numbers of the gene and the production of more salivary amylase enzyme. Copy number variation in $A M Y 1$ was then quantified in multiple world populations, some with high-starch diets and some with low-starch diets. Populations with high-starch diets were found to have, on average, higher copy numbers of $A M Y 1$. Comparisons with other loci in a subset of these populations suggest that the level of $A M Y 1$ copy number differentiation is unusual.

Conclusions: Both chimpanzees and humans have nontaster variants at the TAS $2 R 38$ gene that have independent origins and have likely been maintained within species by balancing selection. This suggests an adaptation linked to the consumption of plants with such chemicals. Our investigation of $A M Y 1$ gene copy number variation in humans indicates that higher copy number in populations with highstarch diets is due to positive selection. A high-starch diet is a characteristic of agricultural societies and hunter-gatherer groups in arid environments. Other hunter-gatherers as well as pastoralists typically eat starch to a much lesser extent. One of the major selective forces may be during childhood, when diarrheal diseases are a major cause of death and, in populations that consume more starch, having higher 
AMY1 copy number may allow a child to receive more nutrients through greater starch digestion starting in the mouth during these diarrheal episodes. The advent of agriculture resulted in major changes to our diet (i.e., a much higher intake of starches in agricultural populations), and increased copy number of this gene appears to have been a rapid means of adapting to this new environment. This example of positive selection on a copy number variable gene is one of the first in the human genome.

\section{A5 \\ Copy Number Variation, Purified Eicosapentaenoic Acid, and Central Nervous System Disorders}

Mehar S. Manku

Amarin Pharmaceuticals Ltd., The Oxford Science Park, Oxford, UK

Objectives: Ultrapure ethyl-eicosapentaenoic acid (ethyl-EPA) is a semisynthetic, ethyl ester of a highly purified form of the fatty acid eicosapentaenoic acid (all-cis-5, 8, 1 4, 17-icosapentaenoic acid [EPA]). The ethyl group is cleaved from the EPA in the gut, and the EPA is absorbed into the bloodstream. Although the exact mechanism of action of ethyl-EPA in Huntington's disease (HD) is not known, it is thought to stabilize mitochondrial integrity and improve neuronal dysfunction. EPA exerts multiple biological activities, supported by both in vivo and in vitro studies and also by placebo-controlled clinical trials. A few biological effects of EPA are cardiovascular (antiplatelet effects) and lowering of triglycerides, for which it is approved medicine in Japan; in rheumatology and inflammation, it acts as an anti-inflammatory; and in oncology EPA is used as an anticancer agent and exhibits anticachexia activity. However, most of the studies carried out to date are on mixtures of EPA and docosahexaenoic acid (DHA). With the availability of pure EPA as an ethyl ester, we have concentrated our efforts on investigating the effects of EPA in the field of psychiatry and other CNS disorders.

HD is a devastating disease of the brain, which usually begins between the ages of 30 and 50 years and progresses to death within 15 to 25 years. It is inherited as an autosomal dominant disease and is associated with increases in the length of a cytosine adenosine guanine (CAG) triplet repeat present in a gene located on chromosome 4p16.3. HD patients have from 36 to over $100 \mathrm{CAG}$ repeats, although most patients have a range 30 to 60 repeats. The greater the number of $\mathrm{CAG}$ repeats, the earlier the onset of the disease. Once symptoms begin, the number of CAG repeats has a modest influence on the rate of progression. Evidence suggests that repeat lengths of 42 to 44 are associated with later progression to late-stage disease. All individuals who inherit the gene develop the disease and eventually die. A number of different rating scales have been used for evaluation of disease progression. The most useful features of these have been combined into the Unified Huntington's Disease Rating Scale (UHDRS), which is now widely accepted as the main evaluation instrument to be used in clinical trials. This scale assesses four main components: motor function, cognition, behavior, and functional abilities. Total Motor Score-4 (TMS-4) of the UHDRS is used to assess motor function.

Results: Briefly, 135 patients were randomized to receive ethylEPA 2 g per day or placebo. In the intention-to-treat population, there was no significant difference in TMS-4 changes from baseline between ethyl-EPA- and placebo-treated patients after either 6 or 12 months of treatment. However, on investigation of the effect of CAG repeat on outcome, there was a significant $(p=0.03)$ improvement in TMS-4 on ethyl-EPA at both 6 months $(-16.9 \%)$ and 12 months $(-15.2 \%)$ in patients with 44 or fewer CAG repeats. High-resolution MRI cerebral scanning was carried out at baseline, 6 months, and 1 year in 30 patients with stage I or II HD who took part in a randomized, double-blind, placebo-controlled trial of $2 \mathrm{~g}$ daily ethyl-EPA. Treatment with ethyl-EPA was associated with significant reduction in brain atrophy in HD, particularly in the head of the caudate and the posterior thalamus.

Conclusions: Further clinical and neuroimaging studies are required to understand the relationship of CAG repeat length with clinical progression in HD and developing medicines that are most suited to CAG variations. Omega 3 fatty acid EPA, via its pleiotropic modes of action, offers a therapeutic benefit in the treatment of early stages of HD.

\section{Session II: Frontiers in Nutrigenetics (continued)}

\author{
A6 \\ FTO Variants Are Associated With \\ Overeating and Many Long-Term Health \\ Problems Associated With Obesity \\ Colin N.A. Palmer \\ Population Pharmacogenetics Group, Biomedical \\ Research Institute, University of Dundee, Ninewells \\ Hospital and Medical School, Dundee, Scotland, UK
}

Monogenic obesity disorders and mouse models of obesity have overwhelmingly pointed to hyperphagia as the predominant genetic force in promoting obesity. Therefore, it was no surprise when the most robust common variant associated with obesity from genomewide association studies was associated with increased energy intake. We and other groups have shown that variants in the FTO gene are not associated with increased food intake per se but rather display a preference for energy-dense foods resulting in an increased calorific intake. This leads to an increase in body mass index (BMI) that is readily detectable in children as young as 4 years of age. This increase in BMI is largely due to an increase in whole-body fat mass, and specific fat depots are not selectively involved in this phenotype. Mice deleted for the FTO locus display hyperphagia; however, increased metabolism leads to a leaner phenotype, whereas in humans, increased metabolism also is observed in the hyperphagic minor allele carriers, but this is clearly not sufficient to prevent obesity in these individuals. This increased BMI from early childhood to adulthood leads to an increased risk of type 2 diabetes, dyslipidemia, and hypertension and finally results in premature death through cardiovascular disease. These observations represent a clear Mendelian randomization experiment confirming the powerful causal role of poor dietary habits and 
obesity in driving the premature morbidity and mortality that is observed in many populations around the world.

\section{A7 \\ Genotype-Dependent Response to Energy-Restricted Diets in Obese Subjects}

\section{J. Alfredo Martínez \\ Institute of Food and Nutritional Sciences, University of Navarra, Pamplona, Spain}

Background and Aims: The excessive fat accumulation occurring in obesity is the result of a disequilibrium between energy intake and expenditure, which partly depends on genetically regulated processes affecting appetite, adipogenesis, thermogenesis, adipocite differentiation, etc. In this context, the response to energy-restricted diets by obese individuals also may be affected by inherited-mediated mechanisms, whose characterization may contribute to a more precise diagnosis and personalized, nutritionally based treatments. The aim of this contribution is to provide evidence about identified genetic polymorphisms with a role in the interaction between genes and nutrition concerning weight loss and metabolism in obese individuals.

Methods: Updated controlled intervention studies selected from the past 10 years concerning candidate genes, putatively involved in body weight homeostasis and metabolism, were critically assessed in relation to their potential participation in weight changes as affected by the diet. The associated phenotypes and variables that were specifically screened included body or BMI changes, fat or regional adipose tissue losses (\%), satiety, and metabolic improvements after the intervention trial.

Results: Clinically relevant evidence has been reported about the involvement of different SNPs concerning genes regulating food intake (MC4R, LEP, LEPR, POMC, FTO, etc.) and fat metabolism and thermogenesis (PPARG, ADBRs, UCPs, etc.), inflammation, and signaling (IL-6, ADIPOQ, CD36, etc.), which produced different responses to energy-restricted diets or macronutrient content (fat, fiber, etc.) of the dietary treatment concerning weight loss, along with metabolic benefits on insulin sensitivity, lipid markers, satiety, etc.

Conclusions: Different nutritional strategies based on energy restriction or macronutrient distribution manipulation devised to induce weight/fat loss were differentially effective depending on the genotype of the obese individual being treated.

\section{A8 \\ The Genetics of Hypertension and Salt Sensitivity}

Steven C. Hunt

Department of Cardiovascular Genetics, The University of Utah, Salt Lake City, Utah, USA

Animal models have unequivocally shown that hypertension follows the kidney. There is also evidence for differential, possibly additive, influences of central versus kidney-specific hormonal blood pressure control of salt balance. In any homeostatic system such as salt balance, multiple factors are involved in counteracting any factor that perturbs the system, forcing it out of balance. These compensating factors, if working efficiently, should return the system back to balance. Should environmental or genetic effects prevent appropriate compensation over the long term, hypertension will likely develop. However, there are also likely to be genetic initiating factors that would lead to hypertension if they are not adequately compensated and that may be strong enough on their own so that complete compensation is not attained. When studying the genetics of these initiating factors, associations will be masked by the degree of compensation and perhaps not even found if compensation is nearly complete. Detecting the genetic initiators may require studying associations after acute interventions and prior to long-term compensation. Detection also may depend on the genetic backgrounds of the subjects being studied: Subjects with few hypertension genes may show little association with any particular gene, whereas subjects with many hypertension-susceptibility genes may show strong associations.

Although some genes have been consistently related to elevated blood pressure and hypertension, the observed effects of these genes are small and hard to replicate in all studies. These common genes almost always have been related to renal electrolyte handling, similar to mechanisms of the rarer monogenic hypertension disorders. Several large studies now have the power to detect hypertension genes with smaller effect sizes and to assess interactions with diet and other environmental risk factors for hypertension. Intervention studies appear to magnify the baseline effects of genes so that they are more easily detected. In addition to genetic interactions with dietary salt on blood pressure, there appear to be important but less understood genetic interactions with dietary fat and cholesterol on blood pressure pathways. Multiple interventions-including less dietary salt, increased dietary potassium, increased fruits and vegetables, lower fat intake, weight loss, and drug treatment - appear to help reduce blood pressure to a greater extent in subjects genetically susceptible to hypertension than those not as susceptible. As one would hope, it appears that those at highest genetic risk of hypertension compared with those at low risk show a greater improvement in blood pressure for interventions that target the defective genetic pathways. There remains an urgent need for the addition of dietary and pharmacologic interventions to genetic studies and vice versa, so that biological mechanisms may be uncovered represented by these statistical interactions and additional interactions discovered. Knowledge arising from such studies may be used to design specific dietary, exercise, weight loss, and drug interventions for the subset of patients that will benefit the most from that intervention. 


\section{A9 \\ Physiological Significance of SNPs: The Folate Example}

\section{Cornelia M. Ulrich}

Fred Hutchinson Cancer Research Center, Seattle, Wash., USA, and German Cancer Research Center (DKFZ), Heidelberg, Germany

Folate is an essential B vitamin utilized for one-carbon transfer reactions, including those related to the methylation of DNA and nucleotide synthesis. Folate deficiency has been linked to a number of diseases, including colorectal cancer, although there may be differences in associations depending on timing, dose, and genetic susceptibility.

Within this metabolic pathway, a large number of genetic polymorphisms, including SNPs and repeats or deletions, have been described. Several of them have been unequivocally linked to relevant biomarkers (e.g., MTHFR C677T, A1298C, and the TS promoter repeat). In addition, some gene-diet interactions within this pathway have been demonstrated and replicated. However, the physiologic significance of multiple polymorphisms in this pathway still has been insufficiently defined.

Our group has completed several epidemiologic and pharmacogenetic studies that illustrate the necessity for considering all functional polymorphisms within a gene simultaneously. In addition, we have developed a mathematical simulation model of folate-mediated onecarbon metabolism (In Silico Metabolism). With this new tool, it is possible to predict the independent and joint effects of multiple genetic variants in this pathway on biomarkers of cancer risk, such as thymidylate synthesis, purine synthesis, and methylation capacity.

\section{A10 \\ Xenobiotic Metabolizing Genes, Meat Intake, and Risk of Advanced Colorectal Adenoma}

\author{
Leah M. Ferrucci ${ }^{1}, 2$, Amanda J. Crosss ${ }^{1}$, Marc J. Gunter ${ }^{3}$, \\ Jiyoung Ahn', Susan T. Mayne'2, Xiaomei Ma², \\ Stephen J. Chanock', Meredith Yeager1, Barry I. Graubard ${ }^{1}$ \\ Sonja I. Berndt', Wen-Yi Huang', Richard B. Hayes4, \\ Rashmi Sinha ${ }^{1}$ \\ ${ }^{1}$ Division of Cancer Epidemiology and Genetics, National \\ Cancer Institute, National Institutes of Health, Bethesda, \\ Md., ${ }^{2}$ Yale School of Public Health, New Haven, Conn., \\ ${ }^{3}$ Department of Epidemiology and Population Health, \\ Albert Einstein College of Medicine, New York, N.Y.; \\ ${ }^{4}$ Division of Epidemiology, Department of Environmental \\ Medicine, New York University School of Medicine, New \\ York, N.Y., USA
}

Objectives: The carcinogenic action of meat-related exposures, such as heterocyclic amines (HCAs), polycyclic aromatic hydrocarbons (PAHs), and N-nitroso compounds might explain the positive associations between the ingestion of red and processed meat and colorectal neoplasia. Single nucleotide polymorphisms in xenobiotic metabolizing enzyme (XME) genes could alter the metabolism of these compounds.
Methods: We evaluated interactions between several XME genes (CYP1A1, CYP1B1, CYP2A6, CYP2C9, CYP2E1, CYP3A4, EPHX1, GSTM1, GSTM2, GSTT1, NAT1, NAT2, NQO1, SULT1A1, and $S U L T 1 A 2)$ and meat-related exposures using a pathway-based approach in 720 cases with advanced colorectal adenoma of the distal colon or rectum and 746 controls from the Prostate, Lung, Colorectal and Ovarian Cancer Screening Trial. Using meat-related databases, we estimated intake of the HCAs 2-amino-3,8-dimethylimidazo[4,5f] quinoxaline (MeIQx) and 2-amino-1-methyl-6-phenyl-imidazo[4,5b]pyridine (PhIP), the $\mathrm{PAH}$, benzo[a]pyrene $(\mathrm{B}[a] \mathrm{P})$, and nitrate/ nitrite.

Results: There were possible interactions between PhIP and CYP1B1 $\left(\mathrm{P}_{\text {interaction }}=0.019\right)$ and NQO1 $\left(\mathrm{P}_{\text {interaction }}=0.007\right), \mathrm{B}[a] \mathrm{P}$ and CYP1B1 $\left(\mathrm{P}_{\text {interaction }}=0.005\right)$ and CYP3A4 $\left(\mathrm{P}_{\text {interaction }}=0.021\right)$, and nitrate/nitrite and CYP1A1 $\left(\mathrm{P}_{\text {interaction }}=0.022\right)$ in relation to colorectal adenoma. However, none of these interactions were statistically significant using a false discovery rate threshold of 0.20 .

Conclusions: Common variants in XME genes may modify the association of HCAs, PAHs, and nitrate/nitrite with advanced colorectal adenoma, but investigation in other populations is required, especially consortia settings.

\section{A11 \\ Genomics of a Slothful Lifestyle: Insights From a Founder Population}

\author{
Alan R. Shuldiner, Toni I. Pollin, Christy Chang, \\ Soren Snitker \\ University of Maryland School of Medicine, Baltimore, \\ Md., USA
}

Obesity and its comorbid consequences such as hypertension, hyperlipidemia, and cardiovascular disease have dramatically increased in prevalence in recent years. Much of this increase has been attributed to positive energy balance due to high-calorie diets and decreased physical activity. However, there is great interindividual variability in response to our slothful lifestyle, with some individuals highly susceptible to obesity and its complications and others protected. Population and family studies suggest that genetics plays a substantial role in determining interindividual variability in response to lifestyle factors, and with the advent of high-throughput, genomewide approaches, variations in specific susceptibility genes and how they interact with lifestyle factors have begun to emerge. The Amish Heredity and Phenotype Intervention (HAPI) Heart Study was initiated to dissect how genes interact with environmental factors to influence cardiovascular disease and related risk factors. From 2002 to 2006, 868 Amish subjects were recruited into the HAPI Heart Study. In addition to baseline cardiovascular traits and physical activity measurements, responses to three short-term interventions were measured, including triglyceride response to a high-fat meal blood pressure response to cold pressor stress and to high/low salt diets and platelet aggregation response to aspirin. Genome-wide association analysis was performed to identify genes/loci associated with these responses. To date, we have identified a nonsense mutation in $A P O C 3$ associated with low postprandial triglycerides and a cardioprotective phenotype, common variants in STK39 (a gene involved in salt homeostasis in the kidney that is associated with blood pressure), and interaction between 
variants in FTO and physical activity on body mass index. These genetic discoveries provide mechanistic insights into gene $\mathrm{x}$ lifestyle interactions that may be useful in providing more personalized diet and lifestyle recommendations to improve health and prevent cardiovascular disease.

\section{A12 \\ FADS2 Polymorphisms Modify the Effect of Breastfeeding on Child IQ}

C.D. Steer ${ }^{1}$, P.M. Emmett ${ }^{1}$ G. Davey Smith², J.R. Hibbeln ${ }^{3}$, J.M. Davis', J. Golding ${ }^{1}$

${ }^{1}$ Centre for Child and Adolescent Health, Department of Community Based Medicine, University of Bristol, Bristol,

${ }^{2}$ Medical Research Council Centre for Causal Analyses in Translational Epidemiology, University of Bristol, Bristol, UK, ${ }^{3}$ National Institute on Alcohol Abuse and Alcoholism, National Institutes of Health, Bethesda, Md., and

${ }^{4}$ Department of Psychiatry, University of Illinois at

Chicago, Chicago, III., USA

Background: A recent study by Caspi et al. has reported that the rs 174575 polymorphism within the FADS2 gene moderates the effect of breastfeeding such that those children with the GG genotype have similar IQs whether breastfed or not. For other genotypes, a clear advantage was seen for breastfed children.

Methods: Data from the Avon Longitudinal Study of Parents and Children were available for 5,934 children with child IQ (WISC III) at age 8 years and genetic data. Genotype data for two polymorphisms (rs174575 and rs1535) were obtained for both the mother and child. Linear regression analysis was used to explore breastfeeding and genetic and interaction effects.

Findings: Breastfeeding was strongly associated with full-scale IQ with a difference of 7.8 points compared to formula-fed infants $(95 \%$ CI $6.6,9.0)$. There was no evidence of a genetic main effect with IQ. An interaction with rs174575 was observed such that breastfed GG children performed better than their formula-fed counterparts by an additional 5.8 points $(1.4,10.1)$ (interaction $\mathrm{p}=0.0091)$. A mother-child genetic interaction was also observed such that GG children of GG mothers performed better than expected by an additional 7.6 points $(2.1,13.0)$ compared to other GG children $(\mathrm{p}=0.0070)$. Similar results were found for rs 1535 and for performance and verbal IQ, although effect sizes were generally reduced for these other outcomes. Interaction results were largely unaffected by adjustment for 7 factors.

Interpretation: This study was unable to replicate the findings of Caspi et al. In contrast to their study, GG children exhibit the greatest difference between feeding methods such that breastfed children performed similarly irrespective of child genotype, whereas formulafed GG children performed worse than other children on formula milk.

\section{Session III: Frontiers in Epigenetics}

\author{
A13 \\ Interindividual Epigenetic Variation: When, \\ Why, and So What?
}

Robert A. Waterland

Departments of Pediatrics and Molecular and Human Genetics, Baylor College of Medicine, and Children's Nutrition Research Center, Agricultural Research Service, U.S. Department of Agriculture, Houston, Tex., USA

Just as interindividual genetic variation contributes to risk of disease, so too can interindividual epigenetic variation. Interindividual variation in DNA methylation and epigenetic regulation has been reported at specific genomic regions, including transposable elements, genomically imprinted genes, and the 'inactive' $\mathrm{X}$ chromosomes in females. Currently, however, we have a very poor understanding of the factors that contribute to interindividual epigenetic variation. In particular, it is important to understand when during the life cycle epigenetic variation arises, why epigenetic regulation varies among individuals, and whether epigenetic interindividuality affects susceptibility to chronic disease. I will summarize current progress toward answering these questions, with an emphasis on recent epigenomic profiling studies that are offering unprecedented insights into the nature and extent of epigenetic variation among humans.

Supported by the National Institutes of Health [5K01DK070007], the March of Dimes Birth Defects Foundation [\#5-FY05-47], and the U.S. Department of Agriculture [CRIS \#6250-51000-049].

\section{A14 \\ Diet and Genomic Modification \\ Alan Jackson, Karen Lillycrop, Graham Burdge \\ Nutrition Biomedical Research Unit, Institute of Developmental Sciences, National Institute for Health Research, University of Southampton/Southampton General Hospital, Southampton, UK}

For the past century, broad social development has been reflected in secular changes in height. There is convincing evidence from population studies that achieved height marks a significantly increased risk for some cancers. Major cancers are associated with increased adiposity, especially with centrally deposited fat for some. Thus, findings of epidemiological studies of the relationship between prenatal growth and risk for specific cancers, metabolic disease, and cardiovascular disease suggest that early-life environment is a causal component in the etiology of these conditions. Mechanistic studies provide some evidence that explains how variations of diet within the normal range of consumption in early life can set later susceptibility through processes such as DNA methylation and covalent modifications to histones. Nutrient 
interventions in laboratory animals during pregnancy and/or lactation show that there is developmental plasticity to environmental stimuli that induces a phenotype that confers survival advantage in the short term but increases susceptibility to pathology in the longer term. These influences can be modified by dietary pattern fed during the weaning period, demonstrating an important interaction between prenatal nutrition and food consumption during later life. This is further implied by the common role for altered epigenetic regulation of specific genes and of altered Dnmt activity. Thus, risk of these seemingly heterogeneous patterns of ill health may reflect a continuum of developmental changes that operate through the same enzymes and pathways that induce epigenetic regulation of specific genes. Risk of specific diseases may reflect the nature and/or magnitude of the environmental exposure during early life. It is not known how these environmental cues may be targeted in a manner that induces altered epigenetic regulation of specific genes or of individual $\mathrm{CpG}$ dinucleotides and so leads to increased risk of different disease processes. However, such specificity is implied by emerging evidence that the magnitude of the maternal nutritional challenge and the relative amount of specific nutrients in the maternal diet induce directionally opposite changes in the physiology and epigenotype of the offspring. Overall, these findings support the concept that a range of prenatal nutritional environments, from constraint to abundance, may induce risk of ultimate different pathological processes. The induced epigenetic changes are likely to be permissive for altered gene expression and hence determine the interaction between an organism and its environment over the life course and, in turn, determine whether increased risk due to the early-life environment becomes disease in later life.

\section{A15 \\ The New Epigenetics: Adapatability and Diversity Through Fidelity}

\author{
R. Lane ${ }^{1}$, R. McKnight ${ }^{1}$, L. Joss-Moore ${ }^{1}$, X. Ke ${ }^{1}$, O. Fu', \\ K. Aagaard², K. Grove 3 \\ ${ }^{1}$ Division of Neonatology, Developmental Origins of Health \\ Laboratories, Department of Pediatrics, The University of \\ Utah, Salt Lake City, Utah, ${ }^{2}$ Division of Maternal Fetal \\ Medicine, Department of Obstetrics and Gynecology, \\ Baylor College of Medicine, Houston, Tex., ${ }^{3}$ Oregon \\ National Primate Research Center, Oregon Health \& \\ Science University (OHSU), Beaverton, Oreg., USA
}

Objectives: Human data convincingly demonstrate that exposure to early-life stresses affects postnatal health by reprogramming gene expression. A mechanism that plausibly links early-life stresses to reprogramming is epigenetics. Studies in our laboratory initially demonstrated that perinatal stresses such as malnutrition affect genome-wide epigenetic characteristics such as DNA methylation and histone acetylation. Subsequent studies demonstrated that these events affect the epigenetic characteristics of specific genes in locus-, tissue-, and gender-specific manners. However, the vast majority of these studies were performed in rodent models of perinatal stress, and the question remains whether these early-life events could potentially reprogram the primate genome. This is particularly important when considering the differences in gestational length and placentation in primates versus rodents.
Methods: We utilized tissue from the previously characterized model of a maternal high-fat diet in a nonhuman primate (NHP) pregnancy, pioneered by Kevin Grove from OHSU. Pregnant animals in this study are allowed free access to a high-fat and calorie-dense diet. Tissues studied include liver and hypothalamus because of the relevance in terms of metabolic homeostasis and appetite regulation. The expressions of genes such as the glucocorticoid receptor (GR) and its isoforms were determined. Genome-wide epigenetic characteristics and the epigenetic characteristics of other specific genes also were determined.

Results: Like rodent models of perinatal malnutrition, the maternal high-fat diet affected genome-wide epigenetic characteristics in the NHP liver. More importantly, like rodent models, the maternal high-fat diet affected the expression of specific genes, such as the GR, in the NHP liver and hypothalamus. Specifically, the high-fat diet affected hypothalamic GR isoform expression in the hypothalamus, which is important because of the potential affects on GR signaling and the relationship between variations in isoform expression and gene-specific epigenetic characteristics.

Conclusion: The molecular machinery of the fetus and neonate is sensitive to stressful environments. Often, the changes occur in regions other than the promoter. Furthermore, many of the epigenetic consequences of perinatal stress are gender and tissue specific. The majority of these changes appear to allow immediate adaptation but lead to postnatal diseases such as diabetes and obesity. A key characteristic of the epigenetic response that we observe across species and diseases is the ability to adapt through responses that are specific not only to the environment but also to gender and tissue.

\section{A16 \\ Choline: Its Interaction With SNPs and Epigenetic Control}

Steven H. Zeisel

The University of North Carolina at Chapel Hill, Chapel Hill, N.C., USA

In a National Institutes of Health (NIH)-funded study, we are determining the human dietary requirements for choline. Subjects are hospitalized in a research facility and are fed carefully defined diets in which we remove choline, and we determine whether they develop liver or muscle dysfunction (using MRI and clinical blood tests) that reverses when we restore dietary choline. We found that most men and postmenopausal women developed organ dysfunction on low choline, but only $40 \%$ of young women did. We discovered that the gene in liver that is responsible for producing choline from scratch, PEMT, has a promoter sequence (switch) in front of it that is turned on by estrogen. We identified the DNA coding sequence for this switch and are currently asking whether phytoestrogens and drug ana$\operatorname{logs}$ of estrogen can induce this gene. Using our human studies, we discovered that there are very common single nucleotide polymorphisms (SNPs) (gene misspellings) that make humans require more dietary choline. One of these SNPs is in the gene PEMT and prevents estrogen from inducing the gene. This SNP occurs in more than half the population. Another SNP in folic acid metabolism causes an increased demand for choline and thereby increases by 85 -fold the likelihood that a person will become sick when deprived of choline. 
We are currently examining whether there are copy number variations in these important genes of choline metabolism. We are collaborating in a number of epidemiology studies that examine the relationships between diet, these gene SNPs, and risk for breast cancer, colon cancer, birth defects, and heart disease. The Zeisel team, after identifying an SNP of interest, makes a knockout mouse model with which to further study phenotypes that might occur in humans. We now have three such knockouts. One of them develops mitochondrial abnormalities and has immotile sperm. We plan studies in humans to see whether SNPs in this gene result in male infertility.

In another NIH-funded study, the Zeisel team examines choline's role in brain development. We know that, in mice and rats, extra choline fed to pregnant dams results in offspring that are smarter on maze testing. We discovered that the progenitor cells that become neurons in brain hippocampus (memory center) proliferate faster if they are exposed to more choline; at the same time, they die slower by apoptosis. One explanation may be that choline changes epigenetic marks on DNA, thereby changing the expression of genes regulating cell division and apoptosis. We found that, indeed, changing dietary choline in pregnant mice changes DNA and histone methylation in neuronal stem cells of fetal brain and that this directly alters cell cycle. We just discovered that the same is true for endothelial stem cells in fetal brain, thereby altering angiogenesis (blood vessel formation). The Zeisel team is currently exploring an alternative hypothesis: that PEMT in fetal brain is very active and that it is the major route for incorporating docosahexaenoic acid into brain. This essential fatty acid is known to be needed for brain development. We are starting new projects to examine whether choline influences development of the retina and to determine whether choline can change rates of stemcell proliferation in adult brain.

\section{A17 \\ Dietary Polyphenols Mediate Regulation of Oxidative Stress and Chromatin Remodeling in Inflammation}

\section{Irfan Rahman \\ Department of Environmental Medicine, University of Rochester Medical Center, Rochester, N.Y., USA}

The therapeutic benefits of fruits, vegetables, tea, and wine are mostly attributed to the presence of phenolic compounds. Naturally occurring dietary polyphenols such as curcumin (diferuloylmethane, an active component of the spice turmeric) and Resveratrol ${ }^{\mathrm{TM}}$ (phytoalexin, a flavonoid found in red wine) can directly scavenge ROS and modulate signaling pathways mediated via NF-кB and MAP kinase pathways and upregulate glutathione/phase II enzyme biosynthesis gene via activation of Nrf2. They also downregulate the expression of proinflammatory mediators, matrix metalloproteinases, adhesion molecules, and growth factor receptor genes by inhibiting histone acetyltransferase activity and activating histone deacetylase (HDAC)/sirtuins (SIRTs). The expression of NF-кB-dependent proinflammatory genes in response to oxidative stress is regulated by the acetylation-deacetylation status of histones bound to the DNA. It has been reported that, in severe asthma and in chronic obstructive pulmonary disease (COPD) patients, oxidative stress not only activates the NF-кB pathway but also alters the histone acetylation and deacety- lation balance via posttranslational modification of HDACs. Corticosteroids have been one of the major modes of therapy against various respiratory diseases such as asthma and COPD. Failure of corticosteroids to ameliorate such disease conditions has been attributed to their failure to either recruit HDAC2 and SIRT1 or to the presence of an oxidatively/posttranslationally modified HDAC2/SIRT1 in asthmatics and COPD patients. Dietary polyphenols such as curcumin, Resveratrol ${ }^{\mathrm{TM}}$, and catechins have been reported to modulate epigenetic alterations in various experimental models. The antiinflammatory property of curcumin, Resveratrol ${ }^{\mathrm{TM}}$, and catechins may be due to their ability to induce HDACs/SIRT1 activity and thereby restore the efficacy of glucocorticoids or overcome its resistance. Thus, these polyphenolic compounds have value as antioxidant, anti-inflammatory, and adjuvant therapies with steroids against chronic inflammatory, epigenetically regulated diseases. The current knowledge on the mechanism of action of these polyphenols in the light of deacetylases in regulation of chromatin remodeling in inflammation will be presented.

\section{A18 \\ Dietary Manipulaton of Histone Structure and Function}

Emily Ho

Oregon State University, Corvallis, Oreg., USA

The classic view of cancer etiology is that genetic alterations (via genotoxic agents) damage DNA structure and induce mutations, resulting in nonfunctional proteins that lead to disease progression. More recently, the role of epigenetic alterations during cancer has gained increasing attention and has resulted in a paradigm shift in our understanding of mechanisms leading to cancer susceptibility. These features are potentially reversible and may affect genomic stability and expression of genes, including tumor-suppressor genes and oncogenes. Aberrant epigenetic events such as DNA hypermethylation and altered histone acetylation have been observed in prostate cancer. The reversible acetylation of histones is an important mechanism of gene regulation. A balance exists in normal cells between histone acetyltransferase (HAT) and histone deacetylase (HDAC) activities, and when this balance is disrupted, cancer development can ensue. During prostate cancer progression, specific modifications in acetylation patterns on histones are apparent. HDAC activity increases in metastatic cells compared with normal prostate, and global changes in acetylation pattern predict prostate cancer risk and recurrence. Targeting the epigenome, including the use of HDAC inhibitors, is a novel strategy for cancer chemoprevention. Recently, drugs classified as HDAC inhibitors have shown promise in cancer clinical trials. We have found that sulforaphane (SFN), a compound found in cruciferous vegetables, inhibits HDAC activity in human colorectal and prostate cancer cells. Based on the similarity of SFN metabolites and other phytochemicals to known HDAC inhibitors, we have found that SFN acts as an HDAC inhibitor in the prostate, causing enhanced histone acetylation, derepression of $P 21$ and $\mathrm{Bax}$, and induction of cell cycle arrest/ apoptosis, leading to cancer prevention. The ability of SFN to target aberrant acetylation patterns, in addition to effects on phase 2 enzymes, may make it an effective chemoprevention agent. Other dietary agents such as butyrate, allyl sulfides, and organoselenium 
compounds also have shown promise as HDAC inhibitors. These studies are also significant because of the potential to qualify or change recommendations for high-risk prostate cancer patients and thereby increase their survival through simple dietary choices incorporating easily accessible foods into a patient's diet and will provide a strong scientific foundation for future large-scale human clinical intervention studies.

\section{Session IV: The Impact of Transcriptomics on Nutrigenomics}

\section{A19 \\ Nrf2 Mediates Cancer Protection but Not Longevity Induced by Caloric Restriction}

Kevin J. Pearson, Rafael de Cabo

University of Kentucky College of Medicine, Lexington, Ky., USA

Caloric restriction (CR) has long been established as an effective system of tumor prevention and has been shown to significantly decrease tumor formation in a wide variety of spontaneous and induced forms of cancer, including chemically induced skin tumors. The involvement of detoxification and antioxidant genes in the prevention of carcinogenesis induced by free radicals is receiving increased research attention. Nuclear factor E2-related factor 2 (Nrf2), the primary transcription factor responsible for mediating these antioxidant cellular signaling cascades, has been shown to play a pivotal role in tumor initiation through its disassociation from Keap- 1 and its nuclear localization in response to chemopreventive phytochemicals. Initially, we found that CR activated Nrf2 and increased levels of several of its downstream effectors, including NAD $(\mathrm{P}) \mathrm{H}$-quinone oxidoreductase 1 , glutathione $\mathrm{S}$-transferases, and heme oxygenase 1 . We then used Nrf2-disrupted (KO) mice to examine this transcription factor's role in the enhanced protection against chemical-induced carcinogenesis, reduced insulin/insulin-like growth factor 1 signaling, and prolongevity effects seen in response to CR. Nrf2-KO mice developed tumors more readily in response to carcinogen exposure than did wild-type (WT) mice, and CR was ineffective in suppressing tumors in the $\mathrm{KO}$ mice. However, CR was capable of improving insulin sensitivity and extending lifespan in both KO and WT mice. Our data suggest that the lifespan extension seen in CR mice is not solely a consequence of upregulation of the $\mathrm{Nrf} 2$ pathway and dissociates the prolongevity and anticarcinogenic effects of CR.

\section{A20 \\ Dietary and Lifestyle Interventions Alter Human Gene Expression}

Per-Arne Svensson

Department of Molecular and Clinical Medicine, Sahlgrenska Academy, Gothenburg University, Gothenburg, Sweden

In addition to its key role in energy storage, adipose tissue is also a major endocrine organ communicating with the brain and peripheral tissues through mediators such as leptin, adiponectin, and other adipokines. Adipose tissue function has been implicated in the development of obesity-related diseases such as diabetes, cardiovascular disease, and cancer. Thus, regulation of genes in adipose tissue may be important in the pathogenesis of obesity and obesity-related diseases. Changes in energy availability have profound effects on adipose tissue functions. We have for several years used expression profiling of human adipose tissue as a tool to gain insights into genes and mechanisms that may be implicated in the development of obesity and related metabolic disease. To this end, the study of expression profiles from adipose tissue during caloric restriction is a valuable tool. However, several challenges exist in the interpretation of expression profiling data. One such challenge is separating the genes regulated directly by caloric restriction from those that are regulated as a result of the reduction in adipose tissue mass. We have used expression information from multiple sources, such as data on various types of dietary interventions, tissue distribution and differences in adipose tissue gene expression between lean and obese subjects, and detailed phenotypic data to facilitate the identification of key genes in obesity. Based on this strategy and information on our well-characterized patient cohorts, we have identified several novel adipocyte-specific genes and genes regulated by weight loss or diet.

\section{A21}

\section{Laboratory Diet Profoundly Alters Gene Expression and Confounds Genomic Analysis}

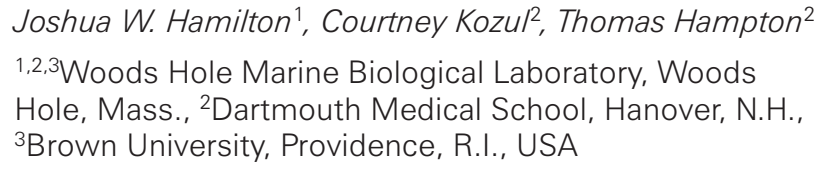

Nutritional studies in laboratory animals have long shown that various dietary components can contribute to altered gene expression and metabolism, but diet alone has not been considered in whole animal genomic studies. In this study, global gene expression changes in mice fed either a nonpurified chow or a purified diet were investigated, and background metal levels in the two diets were measured by ICP-MS. C57BL/6J mice were raised for 5 weeks on either a cerealbased, nonpurified LRD-5001 diet or a purified, casein-based AIN76A diet as part of a larger study examining the effects of low-dose arsenic (As) in the diet or drinking water. Affymetrix Mouse Whole Genome 4302.0 microarrays were used to assess gene expression changes in the liver and lung. Microarray analysis revealed that ani- 
mals fed the LRD-5001 diet displayed a significantly higher hepatic expression of phase I and II metabolism genes as well as other metabolic genes. The LRD-5001 diet masked the As-induced gene expression changes that were clearly seen in the animals fed the AIN-76A diet when each dietary group was exposed to $100 \mathrm{ppb}$ As in drinking water. Trace metal analysis revealed that the LRD-5001 diet contained a mixture of inorganic and organic As at a total concentration of 390 ppb, whereas the AIN-76A diet contained approximately $20 \mathrm{ppb}$. These findings indicate that the use of nonpurified diets may profoundly alter observable patterns of change induced by As and, likely, by other experimental treatments, particularly altering gene and protein expression. This is a particularly important consideration for this and other studies examining the subtle effects of low-level dietary (food or water) As on gene expression and has important implications for other such studies in pharmacology, toxicology, and nutrition that are attempting to investigate the effects of single agents, particularly at low physiological or pharmacological doses.

\section{A22 \\ Toxicogenomic Concepts Applicable to Studies of the Genomic Effects of Dietary Components}

\author{
Karol L. Thompson \\ Center for Drug Evaluation and Research, U.S. Food and \\ Drug Administration, U.S. Department of Health and \\ Human Services, Silver Spring, Md., USA
}

Toxicogenomic analyses are recognized to be of value in assessments of the clinical relevance of adverse events that are observed in animal models. Resources have been developed to help interpret gene expression profiles within the context of a study. Reference compound data sets and pathway mapping tools provide a basis for differentiating pharmacologic from toxicologic effects. From large sets of gene expression data from control groups in toxicogenomic studies, the normal range of variability of individual genes and the contribution of study factors to baseline variability can be assessed. Sources of biological and technical noise can be controlled using performance standards and metrics that have been developed for rat and human samples. These resources, in content or design, have crossover application of interest and utility to nutrigenomic research.

\section{A23 \\ Building the Cairns on the Path to Personalized Medicine}

\section{Lawrence C. Brody \\ National Human Genome Research Institute, National Institutes of Health, Bethesda, Md., USA}

Our understanding of human genetics has advanced significantly over the past two decades. Recent technical advances have enhanced our ability to identify genes involved in disease and have expanded the number of conditions in which these genes can be found. Genes underlying the susceptibility to nearly all common diseases (e.g., diabetes, heart disease, hypertension, common cancers, etc.) have been identified. It is predicted that these findings will advance our understanding of disease in three areas: (1) By identifying the genes involved, we will better understand the biology of disease; (2) genetics will lead to improved diagnostic and prognostic tests and tailored drug treatment; and (3) advances in genetics will usher in an era of preventive medicine based on a person's genomic information. This last application has captured the attention of policymakers, venture capitalists, the media, and the public. It has also ignited a debate within the genetics community about the appropriate use of genetic susceptibility testing for common, complex disease.

In this talk, I will summarize the issues surrounding this debate, describe the state of the science in this area, and present new data from the Multiplex Initiative, a collaborative study aimed at understanding how individuals respond to genetic susceptibility testing for common diseases.

\section{Session V: Noncoding RNAs and Posttranslational Gene Regulation}

\author{
A24 \\ Dietary Regulation of microRNA Expression \\ in Cancer Cells
}

Sharon A. Ross

Nutritional Science Research Group, Division of Cancer Prevention, National Cancer Institute, National Institutes of Health, Bethesda, Md., USA

MicroRNAs (miRNAs) are short ( 18-25 nucleotides), noncoding RNAs that regulate gene expression, predominantly as negative posttranslational regulators, by sequence-specific base pairing in the 3' untranslated regions of the target messenger RNA. Recent work has provided new insights into the role of miRNAs in various biological events, including regulation of cell proliferation, apoptosis, and differentiation processes as well as their dysregulation in cancer and other diseases. In the human genome, recent estimates point to at least thousands of miRNAs. So far, approximately 600 different miRNAs have been described in humans. Studies have demonstrated that there are distinct miRNA expression patterns associated with various tumor sites and that cancer tissues appear to have miRNA expression profiles that are dissimilar from normal tissues. Furthermore, aberrant levels of miRNAs have been associated with tumor progression, invasiveness, metastasis, and acquisition of drug resistance. Human cancers commonly exhibit an altered expression profile of miRNAs with either oncogenic (e.g., miR-21) or tumor-suppressive (e.g., let-7) activity. Only recently have various compounds that affect cell growth and/or differentiation also been shown to affect miRNA expression. Accumulating evidence indicates that dietary components as diverse as folate, retinoids, and curcumin exert cancer-protective effects through modulation of miRNA expression, providing exciting opportunities for the dietary regulation of several biological processes. 
Although the miRNA field has grown tremendously in the past few years, much remains to be discovered, especially with respect to their role in carcinogenesis, as well as potential modification by bioactive dietary components. Issues remain about the downstream targets and pathways affected, the quantity of dietary components needed to bring about a biological effect, the timing of exposure, and other variables that can influence the response. MicroRNAs are likely to be useful as biomarkers of cancer prevention, early disease, or nutritional status, as well as serve as potential molecular targets that are modulated by dietary interventions.

\section{A25 \\ Dietary Methyl Deficiency, microRNA Expression, and Susceptibility to Liver Carcinogenesis}

\author{
I.P. Pogribny, A. Starlard-Davenport, V. Tryndyak, F.A. Beland \\ Division of Biochemical Toxicology, National Center for \\ Toxicological Research, U.S. Food and Drug \\ Administration, Jefferson, Ark., USA
}

Objectives: Altered expression of microRNAs is frequently detected during tumor development; however, it has not been established whether variations in the expression of specific microRNAs are associated with differences in the susceptibility to tumorigenesis.

Methods: Male inbred C57BL/6J and DBA/2J mice were fed a lipogenic methyl-deficient diet that causes liver injury that progresses to liver tumors. Differentially expressed microRNAs were identified by $\mu$ Paraflo microRNA microarray analysis and were validated by quantitative reverse transcription PCR.

Results: We identified 74 significantly upregulated or downregulated microRNAs, including miR-29c, miR-34a, miR-122, miR-155, miR-200b, miR-200c, and miR-221, in the livers of mice fed a methyldeficient diet for 12 weeks compared to their age-matched control mice. The targets for these microRNAs are known to affect cell proliferation, apoptosis, lipid metabolism, oxidative stress, DNA methylation, and inflammation. Interestingly, the $\mathrm{DBA} / 2 \mathrm{~J}$ mice, which develop more extensive hepatic steatosis-specific pathomorphological changes, were characterized by a greater extent of miR-29c, miR34a, miR-122, miR-155, and miR-200b expression.

Conclusions: These results demonstrate that alterations in expression of microRNAs are a prominent fundamental event during the early stages of liver carcinogenesis induced by methyl deficiency. More importantly, our data mechanistically link alterations in microRNA expression to the pathogenesis of liver cancer and strongly suggest that differences in the susceptibility to liver carcinogenesis may be determined by the differences in microRNA expression response.

\section{A26 \\ Alcohol, miRNA Expression, and Its Role in Alcoholic Liver Disease}

\author{
Yueming Tang, Christopher B. Forsyth, Ali Keshavarzian
}

Division of Digestive Disease and Nutrition, Department of Medicine, Rush University Medical Center, Chicago, III., USA

Alcoholic liver disease (ALD) requires endotoxemia and is commonly associated with intestinal barrier dysfunction. MicroRNAs (miRNAs) are recently discovered, small noncoding RNAs that can regulate gene expression by targeting mRNAs and triggering either translational repression or RNA degradation. miRNAs have been implicated in many cellular processes, including cell proliferation, differentiation, apoptosis, and metabolism. Accumulated evidence indicates that aberrant expression of miRNAs is involved in many human diseases, including infection and cancer. We hypothesized that alcohol affects microRNA profile in intestinal epithelial cells and thus induces gut leakiness through negatively influencing synthesis of key tight junctional proteins. To this end, the miRNA expression profile was studied in intestinal epithelial cells from an experimental model of ALD. Male Sprague-Dawley rats were gavaged daily with alcohol $(6 \mathrm{~g} / \mathrm{kg} /$ day $)$ or dextrose as control for 10 weeks. The miRNA profile in the intestinal epithelial cells was analyzed using miRNA microarray (Miltenyi Biotec). We identified that the expressions of miR-212, miR-150, miR142, miR-101, miR-451, miR-96, miR-487, and miR-146 were significantly increased, whereas the expressions of miR-204, miR-494, and miR-183 were significantly decreased by alcohol feeding compared to corresponding controls.

ZO-1 is predicted to be a target gene of miR-212. We previously showed that miR-212 levels in colon biopsy samples in ALD patients were higher than in healthy controls, whereas ZO-1 protein levels were lower. Here, we studied the mechanisms for the involvement of miR-212 in alcohol-induced gut leakiness. We showed that miR-212 is highly expressed in intestinal tissues using a TaqMan microRNA real-time PCR assay. Alcohol-induced miR-212 overexpression is accompanied by reductions in $\mathrm{ZO}-1$ protein expression, disruption of tight junction protein (ZO-1), and increased permeability of Caco-2 cell monolayers. Alcohol-induced miR-212 overexpression correlated with alcohol-induced disruption of monolayer integrity. To demonstrate that miR-212 acts directly at the ZO-1 3'UTR, we inserted the miR-212 target site of ZO-1 3'UTR into luciferase reporter construct and transfected it into Caco-2 cells. The expression of luciferase was significantly decreased when cotransfected with miR-212. This suppression was relieved by a single base mutation in the UTR binding site. To see whether miR-212 regulates ZO-1 levels, we did both overexpression studies using miR-212 precursors and inhibition studies using miR-212-specific antisense oligonucleotide inhibitors (antimiR-212). miR-212 overexpression significantly inhibited ZO-1 protein expression. Knocking down miR-212 expression in Caco-2 cells using anti-miR-212 inhibited alcohol-induced hyperpermeability by $50 \%(\mathrm{p}<0.05)$. Our studies suggest a novel mechanism for alcoholinduced gut leakiness. Alcohol induces miR-212 overexpression, which disrupts intestinal barrier integrity by inhibiting ZO-1 translation. This cascade could lead to dysfunction of tight junction and increase intestinal permeability. This mechanism provides a potential therapeutic target for preventing the leaky gut in patients with ALD. 


\section{Session VI: Moving Beyond Genomics}

\author{
A27 \\ Redox Dysregulation and Oxidative Stress in \\ Schizophrenia: Nutrigenetics as a Challenge \\ in Psychiatric Disease Prevention \\ K.O. Do ${ }^{1}$, P. Conus ${ }^{2}$, J.H. Cabungcal', A. Frank1, R. Gysin'1, \\ S. Lavoie ${ }^{1}$, A. Polari ${ }^{1,2}$, P. Steullet ${ }^{1}$, T. Teichmann ${ }^{1}$, \\ M. Cuenod ${ }^{1}$ \\ ${ }^{1}$ Center for Psychiatric Neuroscience, ${ }^{2}$ Service of General \\ Psychiatry, 1,2Department of Psychiatry; Lausanne \\ University Hospital, Lausanne, Switzerland
}

Background: A developmental dysregulation of glutathione (GSH) synthesis of genetic origin leading to oxidative stress, when combined with environmental risk factors generating reactive oxygen species, can play a critical role in inducing schizophrenia phenotypes. $\mathrm{GSH}$, a major redox regulator and antioxidant, is essential for protection against cellular oxidative damage.

Methods: Reversed translational approach

Results: Patients have decreased GSH levels in cerebrospinal fluid and prefrontal cortex and abnormal GSH synthesis: A GAG trinucleotide repeat polymorphism in the rate limiting GSH synthesizing glutamate-cysteine ligase (GCL) catalytic subunit (GCLC) gene is associated with the disease. The associated genotypes correlate with decreased GCLC mRNA, protein expressions, GCL activity, and GSH content. As demonstrated in various models, such redox dysregulation underlies structural and functional connectivity anomalies and behavioral deficits. In a clinical trial, the GSH precursor N-acetyl cysteine improved both negative symptoms and auditory evoked potentials.

Conclusions: Thus, a genetic GSH synthesis impairment represents one major risk factor in schizophrenia. Redox dysregulation may constitute a "hub" where genetic and environmental vulnerability factors converge, and their timing during neurodevelopment might influence disease phenotypes. The proposed mechanisms should provide biomarkers for early detection, paving the way for prevention perspectives in which nutrigenetics would play a primordial role.

\section{A28 \\ Utilizing Animal Models To Understand Complex Diseases and Dietary Influences: Obesity as an Example}

\author{
Daniel Pomp \\ The University of North Carolina at Chapel Hill, Chapel, \\ Hill, N.C., USA.
}

Most phenotypes displaying continuous variation, including nearly all traits related to energy balance and obesity, are exception- ally complex, with varying contributions of genetic susceptibility and interacting environmental factors. The use of mouse models has been a powerful driving force in understanding the genetic architecture of polygenic traits such as obesity. In addition to the many mouse models of obesity caused by spontaneous mutations and targeted gene knockouts and insertions, the commonly used inbred laboratory strains of mice constitute the primary mammalian model system and are an integral component of obesity research. Within these lines and their derivatives, such as recombinant inbred lines, genome-wide congenic strains, chromosome substitution lines, advanced intercross lines, long-term selection lines, and heterogeneous stocks, there exists a vast array of obesity-relevant genetic and phenotypic variation. The study of such variation, in the form of complex trait analysis including candidate gene analysis and quantitative trait locus (QTL) mapping, has shed significant light on the genetic and genomic architectures of nearly all aspects of energy balance regulation, how body weight and body fat are controlled, and the impact of dietary influences.

Despite these significant efforts, the use of mouse models for the analysis of complex traits such as obesity is at an important crossroad. The use of crosses between inbred strains and/or selection lines has been highly successful in thoroughly populating the mouse obesity predisposition map with QTL for body weight, fatness, energy intake, and energy expenditure, but few if any of these QTLs have been robustly identified at the gene or nucleotide level. At the same time, genome-wide association (GWA) studies in humans have finally overcome many of the power obstacles that had rendered early studies unreliable, and direct identification of human genes for obesity-related traits may call into question the continued use of mice as a model in such efforts. In fact, the success of GWA studies is likely to increase the importance of relevant animal models for several reasons.

First, mouse models will now be important in pursuing the mechanisms of genes discovered in association studies. Second, many important obesity-related phenotypes (e.g., those requiring measures of energy intake and energy expenditure) are challenging for GWA studies because of the high cost of obtaining accurate measurements and require informative animal models for initial evaluation of genetic predisposition. The third, and perhaps the most important, reason for using animal models is the difficulty in implementing robustly powerful designs for human association studies that could test anything beyond relatively simple models of obesity. Appropriately designed animal models can uncover networks of functionally important relationships within and among diverse sets of biological and physiological phenotypes that can be altered by relevant external factors (e.g., diet and exercise) and thus can incorporate multiple genetic, environmental, and developmental variables into comprehensive models describing susceptibility to obesity and its progression.

Such a model is represented by a new paradigm for complex-trait analysis, the "collaborative cross" (CC). The CC is a large panel of recombinant inbred mouse lines derived from a genetically diverse set of eight founder strains. It has a distribution of allele frequencies resembling that seen in human populations, in which many variants are found at low frequencies, and only a minority of variants are common. The eight parental inbred lines contributing to the CC are estimated to capture more than $90 \%$ of the known variation present in all mouse strains. Existing data on the founder strains and on many of the early generations in development of the $\mathrm{CC}$ demonstrate broad variability in many obesity-related phenotypes, indicating that the $\mathrm{CC}$ will represent an excellent resource for identifying genes, controlling 
predisposition to many traits relevant to obesity, and understanding the pathways, networks, and systems that control obesity and how it is impacted by, and interacts with, dietary influences.

\section{A29 \\ Using Pathway Analysis To Go Beyond Simple Genomics}

\section{Chris Evelo}

Department of Bioinformatics, Maastricht University, The Netherlands

Pathway analysis tools such as PathVisio, which was conceptually derived from the well-known GenMAPP program, can greatly assist the understanding of genomics experiments. It allows overrepresentation analysis using, for instance, Gene Set Enrichment statistics, which helps identify which pathways are most affected in a specific experiment. The actual changes in those pathways can then be visualized in a number of ways using a flexible plug-in approach. This plug-in mechanism allows easy development of dedicated new ways to do visualization and analysis.

For optimal pathway analysis, the pathways themselves must be in a usable format. For this purpose, we developed WikiPathways so the scientific community can easily update and curate pathways. A large number of metabolic and regulatory pathways are now available on WikiPathways for many species, and the number and quality of the available pathways increase on a daily basis.

A number of features allow us to use WikiPathways and PathVisio to go beyond simple genomics:

- KEGG and BioPAX importers allow us to use even more pathways from external sources, including Reactome.

- A putative pathway parts interface allows automated suggestions for new pathway entities from text and data-mining results.

- The usage of the BridgeDB software framework for identifier mapping allows us to combine data from different technologies in genomics and metabolomics in a single pathway representation.

- Webservices allows usage of pathway information in other software tools and, for instance, allows combined representation and analysis of new experimental data with published data from ArrayExpress-Atlas (the curated version of the microarray data repository at $\mathrm{EBI})$.

- The same Webservices can be used to analyze data in $\mathrm{R} /$ Bioconductor.

- A Cytoscape plug-in allows representations of pathways such as those in networks. Pathways represented as networks allow combined analysis using data from external data sources about, for example, intracellular locations of gene products, whereas, for instance, representations of multiple pathways connected by genes that were affected in a specific experiment allow us to get a view at an even higher integration level and see what major processes are affected.

The approaches mentioned so far have been extensively used in nutrigenomics analysis in the European Nutrigenomics Organization (e.g., in studies directed toward the development of insulin resistance). I will show some examples of this analysis to illustrate the strength of the approach.
WikiPathways, BridgeDB, and PathVisio are all open-source software projects, freely available from http://www.wikipathways.org, http://www.bridgedb.org, and http://www.pathvisio.org. All three projects are collaborative efforts of the Conklin Lab in San Francisco and the BiGCaT group in Maastricht.

1. WikiPathways: Pathway Editing for the People. Pico AR, Kelder T, van Iersel MP, Hanspers K, Conklin BR, Evelo CTA (2008). PLoS Biol 6(7): e184.

2. Presenting and exploring biological pathways with PathVisio. Martijn P van Iersel, Thomas Kelder, Alexander R Pico, Kristina Hanspers, Susan Coort, Bruce R Conklin, Chris Evelo (2008). BMC Bioinformatics 9: 399 Sep.

3. Mining biological pathways using WikiPathways Webservices. Thomas Kelder, Alexander R Pico, Kristina Hanspers, Martijn P van Iersel, Chris Evelo, Bruce R Conklin. PLoS One 2009 4:7 07.

4. Big data: Wikiomics. Mitch Waldrop. Nature 455:22-25 (2008).

5. We the curators. Allison Doerr. Nature Methods 5:754-755 (2008)

\section{A30 \\ Metabolomics in the Study of Aging and Caloric Restriction}

Bruce S. Kristal, Susan E. Hankinson, Wayne R. Matson, Yevgeniya I. Shurubor, Boris F. Krasnikov, Ugo Paolucci, Honglian Shi, Diane Sheldon, Neil Russell, Matt Sniatynski, Vasant Marur

Department of Surgery and Neurosurgery, Harvard Medical School/Brigham and Women's Hospital, Boston, Mass., USA

Objectives: Dietary or caloric restriction (CR) is the most potent and reproducible known means of increasing longevity and reducing morbidity in mammals. The risk of breast cancer, for example, is generally decreased by more than $90 \%$ in CR rodents, and the CR-mediated effects are usually dominant to those induced by genetic risk factors, carcinogens, or cocarcinogens. The robust observations of reduced morbidity in CR animals are directly analogous to studies in humans that link obesity with poor health outcomes, including increased risk of neoplastic disease. We therefore proposed to test the general concept that biomarkers of diet in rats will predict risk of future disease in humans.

Methods: Metabolomics measurements in sera/plasma were conducted using HPLC coupled with coulometric detector arrays ( $\mathrm{N}=\sim 600$ rats, $\sim 1,700$ humans). Classification and predictive power were tested, optimized, and subsequently validated using a series of megavariate data analysis approaches in sequential blinded cohorts.

Results: Exploratory studies identified 93 redox-active small molecules from sera with the potential to distinguish dietary groups in both male and female rats (60, 6-month-old FBFN1 rats/group, $\mathrm{AL} / \mathrm{CR} / \mathrm{male}$ females in primary set). Partial Least Squares Projection to Latent Structures Discriminant Analysis, a projection method optimized for class separation, built models with $>95 \%$ accuracy in distinguishing groups. Data processing choices of transformation, scaling, and winsorizing (outlier removal) each affected strength of the models and, in some cases, revealed distinct metabolites to be of importance in building these models, often in gender-specific ways. Diets varying in extent and duration of CR were used to develop mod- 
els for intermediate caloric intakes, which are more relevant for human studies (total $\mathrm{N}=\sim 180$ males, 180 females). Markers were adapted for human study, analytically validated at both the instrumentation ( $\mathrm{N}=30 ; 100 \%$ accuracy in blinded splits) and at the sample collection levels $(\mathrm{N}=34$; majority stable under worst-case shipping conditions), then biologically validated $(\mathrm{N}=\sim 200$, metabolites and profiles had intraclass correlation coefficients from $\sim 0.65-0.85)$. We will present these modeling approaches, the models, and their ability to distinguish sera based on caloric intake, as well as data from the initial application of these markers to address risk of breast cancer in case-control studies nested within the Nurses' Health Study.

Conclusions: Metabolomics profiles offer a potential biochemical approach to validate nutritive status and contribute to epidemiological investigations.

\section{Session VII: Frontiers in Nutrigenetics/Nutrigenomics: Building Partnerships}

\section{A31 \\ Opportunities and Challenges for the U.S. Food And Drug Administration}

\section{Claudine J. Kavanaugh}

U.S. Food and Drug Administration, Rockville, Md., USA

The U.S. Food and Drug Administration (FDA) recognizes the importance of genetics in personalized medicine and nutrition. FDA recently has added the Division of Personalized Nutrition and Medicine (DPNM) to develop and implement research strategies that account for the genetic, environmental, and cultural diversity that influences the expression of genetic makeup. Such information is important for improving personal and public health. DPNM has several ongoing projects to evaluate how an individual's genetic makeup may be altered by diet and nutrition. For example, FDA collaborated with the U.S. Department of Agriculture on the Delta Vitamin Project, which evaluated children's genotypes in relation to preserum and postserum vitamin levels in a 3-month summer camp where two nutritious meals were provided. FDA also is leading an effort with other Government agencies, academia, and industry to develop a standardized Web-based database for food and nutrient assessments. FDA recognizes the challenge of balancing nutrition information for the general population with more personalized health messages and is now making this a priority.

\section{A32}

\section{Opportunities and Challenges for the U.S. Department of Agriculture}

\author{
Joseph T. Spence \\ Beltsville Agricultural Research Center, Agricultural \\ Research Service, U.S. Department of Agriculture, \\ Beltsville, Md., USA
}

Food and agriculture are inextricably linked, and that connection too often is overlooked. As part of its mission, the U.S. Department of Agriculture (USDA) conducts research that helps define an optimal and safe diet, enhances agricultural production, and provides dietary guidance based on sound research. Each of these areas will benefit from continued research in the area of nutrigenomics. Recent USDA research in the area of genetic prediction of milk production in dairy cows provides a compelling story of how genomics can very significantly reduce the cost of milk production and greatly enhance the accuracy of the prediction of the best milk producers. The use of genetic prediction provides a threefold increase in the accuracy of the prediction and can be done at $1 / 20$ the cost of traditional methods of analysis. The stunning success of the prediction of dairy production hints at the usefulness of nutrigenomics in human nutrition. This approach is now being used in the production of other agricultural commodities, including legume and other crop production, to increase yield, improve drought tolerance, and develop disease resistance. Although genetic prediction has proven to be successful beyond all expectations, it clearly has changed the dairy industry in both positive and negative ways.

Nutrigenomics no doubt will continue to provide information on metabolic processes and nutritional requirements. Although the possibility of the development of individualized dietary guidance is becoming a reality, there are concerns and challenges. As we develop the capability to identify nutritional requirements for individuals, it will be a challenge to be able to make meaningful changes in a person's diet. Diets are a complex interaction of a variety of foods, nutrients, and nonnutrititive components. Changing one or a number of components of the diet will affect the overall diet in ways that we will not fully understand. The nutritional status of an individual represents not only the recent dietary intake of that individual but also the lifetime of intake of substances in the diet. It is not clear whether the impact of these exposures can be reversed or mitigated. The role of epigenetic influences in influencing the response of an individual to dietary components will prove to be significant and will need to be explored. The expanding importance and impact of nutrigenomics have the potential to raise some challenging ethical concerns. These and other issues will clearly need to be addressed as research in this important area proceeds. 


\section{A33 \\ The Assessment of Diet-Genome Interactions and Their Translation for Public Health Impact: Opportunites and Challenges for the Centers for Disease Control and Prevention}

Renée M. Ned, W. David Dotson, Nicole F. Dowling

Office of Public Health Genomics, National Center for

Chronic Disease Prevention and Health Promotion,

Centers for Disease Control and Prevention, U.S.

Department of Health and Human Services, Atlanta, Ga., USA

Nutrition is an essential element of many public health programs and research, which currently include generalized dietary recommendations, monitoring of the nutritional status of populations through epidemiologic surveys, screening for metabolic disorders in newborns, and identifying dietary risk factors for various disorders and health outcomes. With the availability of quick and inexpensive genotyping technologies, important goals are now to examine gene-environment (diet) interactions, synthesize and interpret these data, and translate the findings for maximal public health impact. Such efforts require population-based research, which lags behind that of basic research. The Centers for Disease Control and Prevention (CDC) conducts large epidemiologic studies, such as the National Health and Nutrition Examination Survey (NHANES), which can be used to monitor dietary and nutritional trends and to assess gene-diet interactions. DNA samples from a subset of NHANES III (1991-1994) and NHANES 1999-2002 participants are available for use by the larger research community. This presents a great opportunity, as NHANES contains a wealth of nutrition data, and few nutrigenetic-related variants have been genotyped to date.

A major obstacle in the translation of public health genetics/ genomics research is the synthesis of available data. The Office of Public Health Genomics at CDC has developed an online tool for knowledge synthesis for use by the nutrigenetics/nutrigenomics field: the HuGE Navigator, a continuously updated and searchable knowledge base of genetic associations and human genome epidemiology. OPHG has also spearheaded two additional mechanisms to advance genomics translation. The first is the Evaluation of Genomic Applications in Practice and Prevention (EGAPP) Initiative, a systematic and evidence-based process for evaluating genetic tests and other health-related applications of genomics technologies. A key goal of EGAPP is to provide credible information that is clearly linked to available scientific evidence that will allow health care providers, payers, consumers, policymakers, and others to distinguish genetic tests that are safe and useful. The second is the Genomic Applications in Practice and Prevention Network (GAPPNet). GAPPNet is a collaborative initiative involving partners from across the public health sector that aims to accelerate and streamline the effective and responsible use of validated and useful genomics knowledge and applications, such as genetic tests, technologies, and family history, into clinical and public health practice. Although nutrigenetic/nutrigenomics research is in its infancy at CDC, various tools and mechanisms are available to help advance the field and to inform public health research, policies, and programs.

\section{A34 \\ Genes, Ariculture, Human Health, and the Climate Issue: Opportunities and Challenges}

\section{Ole Faergeman}

Aarhus University, Aarhus, Denmark

Nuclear war and climate disaster are currently the two great threats to human health and civilization. Although the first is unlikely to happen unless we do something stupid, the second is likely to happen unless we do something smart. Twenty years ago, in an article for fellow physicians about the potential health effects of global climactic and environmental changes, Alexander Leaf wrote that "we have been educated and given a mandate by society to be the guardians of its health. When issues portend such disastrous consequences to health, we must make them our concern." Some medical societies have now, finally, done so.

Physicians and other professionals, not least researchers in medical and nutritional sciences, know enough about chemistry, physics, statistics, and other disciplines to read the climatological literature and to understand how the climate problem is related to a range of different issues, including disease, nutrition, and the food industry.

I shall argue that climate change is related to human disease in two ways. Diseases such as malnutrition, vector-borne infectious diseases, thermal stress, injuries, and psychiatric illness are anticipated to become more common as the earth surface warms and the climate becomes less stable. They are a consequence of climate change, and health systems all over the world must adapt to these new disease patterns. Dealing with them is thus part of the strategies for adapting to climate change.

Other diseases such as respiratory illness, type 2 diabetes, obesity, coronary artery disease, and certain cancers are not caused by climate change. Instead, they share causes with climate change. Burning fossil fuels and other fuels produces not only greenhouse gases but also particulate pollution causing respiratory illness. Raising livestock accounts for about $18 \%$ of anthropogenic greenhouse gases, and eating large amounts of red meat is related to death by cancer and cardiovascular disease. Leaving oil and coal in the ground and reducing the production of livestock thus help mitigate climate change and at the same time enhance human health.

Farmers, scientists, and physicians are necessarily engrossed in the quotidian details of producing food, understanding nutrition, and dealing with diseases of malnutrition and hypernutrition. Indeed, many of them do so at the level of plant, animal, and human genetics. Climate change relates these different fields ominously to each other, however, and each of us should feel encouraged to participate in the greater public debate about what to do to avert the "disastrous consequence to health" that Leaf described. We can do so with a point of departure in the farmyard, the laboratory, or the clinic.

1. Leaf A. Potential health effects of global climatic and environmental changes. The New England Journal of Medicine 1989;321:1577-1583. 


\section{A35}

\section{Building Industry-Academia Partnerships}

Peter J. Gillies ${ }^{1}$, Penny Kris-Etherton ${ }^{2}$

${ }^{1}$ Applied Biosciences, Newark, Wilmington, Del., ${ }^{2}$ The

Pennsylvania State University, University Park, Pa., USA

In an era of open innovation, the intersection of industry and academia affords a special space to integrate collective knowledge, experience, and technology to produce healthier foods and dietary supplements. Fueled by nutrigenomics, such products may exhibit unsurpassed nutritional pharmacology based on integrated nutrientgene interactions.

Unfortunately, the interactions of industry and academia are sometimes viewed with bias, suspect science, and conflicts of interest. Human frailties notwithstanding, relationships based on shared ethical values, rigorous science, carefully selected projects, respect for institutional boundaries, and attention to the "optics" of the relationship can lead to success. Based on almost a decade of history between DuPont and The Pennsylvania State University and using omega-3 fatty acid research as an heuristic example, a productive industry-academic partnership model in molecular nutrition will be presented along with a discussion of the recent recommendations promulgated by the International Life Sciences Institute North America Working Group on Guiding Principles related to industry-academia funding practices in nutrition science.

\section{A36}

\section{Tailoring New Zealand Foods To Match} People's Genes

Lynnette R. Ferguson ${ }^{1,3}$, Rong Hư ${ }^{1,3}$, Wen Jiun Lam ${ }^{1,3}$, Karen Munday ${ }^{1,3,4}$, Christopher M. Triggs ${ }^{2,3}$

${ }^{1}$ Discipline of Nutrition and ${ }^{2}$ Department of Statistics, The University of Auckland, Auckland, New Zealand;

${ }^{3}$ Nutrigenomics New Zealand; ${ }^{4}$ Institute of Food, Nutrition and Health, Massey University, Palmerston North, New

Zealand

Nutrigenomics New Zealand is tasked with developing a competency that could be utilized by the food industry to develop gene-specific personalized foods. On strict definitions, this program is one of nutrigenetics. A considerable amount of published work involves the response of a single gene, usually in the form of a single nucleotide polymorphism (SNP), to a single nutrient. We are utilizing inflammatory bowel diseases, in particular Crohn's disease (CD), as proof of principle, taking a tiered approach that begins and ends with the human population. Knowledge of key human disease SNPs is incorporated into the design of paired reporter gene constructs whereby isogenic cell lines, with and without the variant SNP of interest, are tested for phenotypic effects of nutrients, bioactive compounds, and food extracts. Lead compounds then are tested in relevant animal models before developing into foods. In parallel, we are testing New Zealand CD and control populations for key genetic variants and comparing this information with a detailed dietary analysis. Such an approach led to the identification of mushroom intolerance in individuals carrying variants in an organic cation transporter gene (OCTN1), whereas a range of different foods is associated with the more common variants in the main CD gene, NOD2. Merging understanding of the complexity of the human genome reveals the importance of more complex DNA alterations such as copy number variants and epigenetic events in regulating the expression of genes. Interactions, including gene-gene, gene-diet, and gene-environment, add further complexity. A substantial component of the program relies on high-quality data management, bioinformatics, and biostatistics. International linkages will be essential for the enhanced success of this program.

Acknowledgments: Nutrigenomics New Zealand (www.nutrigenomics.org.nz) is a collaboration between AgResearch Limited, Crop \& Food Research, HortResearch, and the University of Auckland and is largely funded by the Foundation for Research, Science and Technology. 


\section{Poster Presentations}

\section{Journal of \\ Nutrigenetics \\ Nutrigenomics}

\section{P1 \\ Thrombospondin 1 Secretion and Expression Are Affected by Glucose in Primary Cultured Adipocytes From High-Fat-Diet-Fed Animals}

\author{
A.V. Arellano, D.F. Garcia-Diaz, J. Campion, F.I. Milagro, \\ J.A. Martinez \\ Unversity of Navarra, Pamplona, Spain
}

Objectives: To investigate if the secretion and expression patterns of thrombospondin 1 (TSP-1), a well-known antiangiogenic factor and TGF- $\beta$ activity regulator, are affected by insulin or high/low glucose concentration on primary cultured adipocytes from obese and lean rats.

Methods: Adipocytes from epididymal adipose tissue from male Wistar rats, which were fed either on a standard $(\mathrm{C} ; \mathrm{n}=10)$ or a highfat diet $(\mathrm{HF} ; \mathrm{n}=10)$ for 50 days, were isolated and then incubated with high (HG; $25 \mathrm{mM})$ and low (LG; $5.6 \mathrm{mM})$ glucose concentrations, in the presence or absence of $1.6 \mathrm{nM}$ insulin for 24 to $72 \mathrm{~h}$. Also, different obesogenic parameters were analyzed, including TSP-1 serum levels. At the end of the in vitro experimental assay, TSP-1 secretion to culture medium at $72 \mathrm{~h}$ was analyzed, and TSP-1 gene expression levels at 0,24 , and $72 \mathrm{~h}$ were measured by RT-PCR. In addition, glucose uptake and glycerol release were also determined at $24 \mathrm{~h}$ and $72 \mathrm{~h}$.

Results: Rats fed on an HF diet showed overweightness compared to controls, including increased adiposity, hyperleptinemia, and hyperglycemia phenotypes. Serum TSP-1 levels and TSP-1 mRNA basal expression of adipocytes from HF-fed rats presented higher levels than controls, although without reaching statistical significance. TSP-1 secretion in culture after $72 \mathrm{~h}$ showed a significant interaction between the previous dietary intake and glucose incubation, mainly induced by a dramatic rise in TSP-1 secretion from HF diet-HG treated adipocytes. Surprisingly, mRNA analysis did not match with TSP-1 secretion changes, presenting higher expression levels in HF diet-LG treated adipocytes at $24 \mathrm{~h}$, followed by a remarkable decreased expression on this group at $72 \mathrm{~h}$. Glucose uptake at $24 \mathrm{~h}$ was modified by glucose concentration in culture and dietary treatments, being higher on $\mathrm{C}$ diet-HG treated adipocytes. At $72 \mathrm{~h}$, glucose uptake was induced by insulin and inhibited by HG treatment and HF dietary treatment. Finally, basal lipolysis, measured as glycerol release, was reduced at both $24 \mathrm{~h}$ and $72 \mathrm{~h}$ in adipocytes from rats fed previously with the HF diet.

Conclusions: TSP-1 has been recognized as an adipokine that correlates with obesity, inflammation, and insulin-resistance processes. It was observed that TSP-1 secretion was highly modified in an in vitro model that mimics insulin-resistance/obesity conditions, showing a higher response to glucose in adipocytes from rats fed on an HF diet. This pattern was opposite to mRNA levels, suggesting a strong posttranscriptional regulation of this gene in adipocytes. The present nutrigenomic analysis confirms an interaction between diet and glucose in adipose tissue regarding this adipokine and reinforces the idea that TPS-1 is closely related with metabolic obesity-related processes.

P2 Changes in Regulation of Metabolic and
Immunological Pathways in Leukocytes Due
to Alterations in Macronutrient Composition Ingerid Arbo ${ }^{1 *}$, Hans-Richard Brattbakk1* , Fedon Lindberg ${ }^{2}$, Bård Kulseng ${ }^{3}$, Mette Langaas ${ }^{1}$, Berit Johansen ${ }^{1}$

${ }^{1}$ Norwegian University of Science and Technology, Trondheim, Norway; ${ }^{2}$ Weight in Balance, Oslo, Norway; ${ }^{3}$ Regional Centre for Obesity Treatment, St. Olav's University Hospital, Trondheim, Norway

Objective: A human intervention study aiming to explore how diet components influence cardiovascular artery disease development and other lifestyle diseases, with inflammation as a common symptom, was performed. We focused on identifying differentially expressed genes in response to a change of diet macronutrient composition between a typical "Western" diet (high in carbohydrate, low in protein and fat) and a balanced diet (equal caloric amounts of carbohydrate, protein, and fat). The project will integrate and further develop several branches of science and technologies, including transcriptomics, proteomics, lipidomics, statistics, bioinformatics, and computational biology — all central aspects of nutrigenomics and systems biology.

Methods: A total of 32 healthy, young, slightly overweight persons went through a two times six days randomized isocaloric crossover diet intervention, fed solely on smoothies with specific macronutrient composition. Fasting blood samples for transcriptome and other biomarker analyses were collected before and after each diet period. Transcriptome profiling was carried out using Illumina HT12 microarrays on leukocyte RNA.

Results: Studying the gene expression after a balanced and a Western diet intervention shows that the macronutrient composition regulates several cellular pathways. These include lipid, carbohydrate, and glycan metabolism pathways, as well as important pathways in the immune system and pathways related to neurodegenerative and infectious diseases.

Conclusion: Changing from a typical Western diet, high in carbohydrate, to a balanced diet, with equal caloric amounts of carbohydrate, protein, and fat, does affect the regulation of metabolic and immunolgical pathways. Dysregulation of these same pathways is commonly connected to the onset of lifestyle diseases.

\footnotetext{
${ }^{*}$ The authors contributed equally to the study.
}

\section{KARGER \\ Fax +41613061234}

E-Mail karger@karger.ch

www.karger.com
(C) 2010 S. Karger AG, Basel

Accessible online at: www.karger.com/jnn 


\section{P3 \\ Changes in Gene Expression and Clinical Measures Associated With the Metabolic Syndrome in Baboons Fed a High Fat, High-Fructose Diet}

\author{
Anthony Comuzzie, Raul Bastarrachea, Maggie Forey- \\ Garcia, Juan Carlos Alveranga-Lopez, Paul Higgins, \\ Michael Proffitt, Elizabeth Tejero, Saroja Voruganti, \\ David Rainwater, Shelley Cole \\ Southwest Foundation for Biomedical Research, San \\ Antonio, Tex., USA
}

A major consequence of the rising prevalence of obesity is the subsequent increased risk for a variety of life-threatening chronic diseases, including type 2 diabetes and cardiovascular disease. At their cores, both of these conditions appear to result from the disruption of normal glucose and lipid metabolism. While there is accumulating evidence that such metabolic dysregulation is strongly influenced by the action of genes, it is becoming increasingly apparent that this impact may be strongly mediated by interaction with environmental factors such as dietary composition. This latter point is of particular importance since diet represents a directly manipulable factor for mediating disease risk. In an effort to evaluate the effects of a diet high in saturated fats and simple carbohydrates in a controlled experimental environment, we provided a high-fat, high-fructose (HFHF) diet to two groups of baboons for a period of 8 weeks. This diet ( $38.5 \%$ calories from fat, $51 \%$ calories from carbohydrates) was formulated to represent the nutritional composition of a typical fast-food meal. One group of animals (HFHF) was provided ad libitum access to this diet and water. The second group $(\mathrm{HFHF}+\mathrm{S})$ also received ad libitum access to this same diet but with the addition of ad libitum access to a drink formulated to represent a typical soft drink along with access to water as well. Animals in the HFHF group showed significant changes in body composition, along with increased LDL levels compared with their baseline values. However, the animals in the $\mathrm{HFHF}+\mathrm{S}$ group showed even a more pronounced response in key metabolic parameters. The $\mathrm{HFHF}+\mathrm{S}$ group showed a significant increase in levels of triglycerides and $\mathrm{HbAlc}$ as well as a significant increase in body fat. The HFHF $+\mathrm{S}$ group also showed a shift toward increasing levels of small dense LDL particles and a decline in HDL levels. Combined, this pattern of physiological markers is consistent with the development of the metabolic syndrome. In addition, the $\mathrm{HFHF}+\mathrm{S}$ group of animals showed a significant drop in adiponectin levels, a condition that has also been reported as characteristic of the metabolic syndrome and that was not observed in the HFHF group. Initial analysis of gene expression by RT-PCR of CNR1, AdipoR1, and AdipoR2 in lymphocytes revealed shifts in expression levels from baseline consistent with the changes observed in the more traditional clinical measures. Therefore, it would appear that exposure to a diet high in both saturated fats and simple carbohydrates can induce characteristics consistent with the metabolic syndrome in baboons in as little as 8 weeks and that this nonhuman primate model of dietinduced metabolic dysregulation could offer a unique opportunity to further explore the interaction of diet and genes in the development of type 2 diabetes and cardiovascular disease.

\section{P4}

Study of Genetic Variation in FTO and MC4R With Food Intake and Body Mass Index in a Mediterranean Population

\author{
Dolores Corella', Paula Carrasco', Carolina Ortega', \\ Olga Portoles², Marisa Guillen², Jose V. Sorli², \\ Oscar Colte//3, Maria Arregui ${ }^{3}$, Ramon Estruch 4 , \\ J.M. Ordovas ${ }^{5}$ \\ ${ }^{1}$ University of Valencia and CIBER OBN, ${ }^{2}$ Valencia \\ University, Valencia, Spain; ${ }^{3} J a m e s$ I University, Castellón \\ de la Plana, Spain; ${ }^{4}$ Clinic Barcelona, Barcelona, Spain; \\ ${ }^{5}$ Human Nutrition Research Center on Aging, Tufts \\ University, Boston, Mass., USA
}

Objectives: Variation in the FTO (fat mass and obesity-associated) and the MC4R (melanocortin-4 receptor) genes has been consistently associated with increased body mass index (BMI) and obesity risk in several populations of different ethnic backgrounds. However, the mechanisms are still unknown, but an effect of both polymorphisms on satiety has recently been suggested. Our objectives were to study the association between the rs 9939609 in the FTO $(\mathrm{T}>\mathrm{A})$ and the rs17782313 ( $\mathrm{T}>\mathrm{C})$ near the MC4R and food consumption and BMI in a Mediterranean population.

Methods: The rs9939609 (FTO) and the rs17782313 (MC4R) were genotyped in two independent Mediterranean samples from Spain, differing in age, BMI, and cardiovascular risk factors. A random sample of adults $(n=1400)$ from the general population (age $42 \mathrm{y}$, BMI: $\left.26 \mathrm{~kg} / \mathrm{m}^{2}\right)$ and a sample $(\mathrm{n}=925)$ of high cardiovascular risk (HCR) elderly subjects (age $67 \mathrm{y} ; \mathrm{BMI}: 31 \mathrm{~kg} / \mathrm{m}^{2}$ ) were studied. Food intake was determined by food frequency questionnaires. Clinical, lifestyle, and anthropometric variables were determined.

Results: Prevalence of rs9939609 (FTO) was 32\%TT, 48\%TA, and 20\%AA; prevalence of rs 17782313 (MC4R) was $61 \% \mathrm{TT}$, $34 \% \mathrm{TC}$, and $5 \% \mathrm{CC}$ with no differences between independent samples. The rs9939609 was significantly associated with BMI in the general population $(\mathrm{P}<0.001)$. Body weight increased with the number of the A alleles (TT: $69+/-13$, TA: $71+/-13$, and AA: $73+/-14 \mathrm{~kg}$; $\mathrm{P}<0.001)$. However, in the HCR sample, no statistically significant association was found (TT: $78+/-13$, TA: $77+/-13$, and AA: $79+/-13$ $\mathrm{kg} ; \mathrm{P}=0.258$ ). The rs 17782313 (MC4R) was significantly associated with BMI in both samples $(\mathrm{P}<0.05)$. TC and CC subjects had $2 \%$ and $8 \%$ higher body weights ( $\mathrm{P}$ for trend $<0.05$ ) than TT subjects. No statistically significant differences in total energy intake or in macronutrient intake were found for any of the polymorphisms in the population as a whole. In subgroup analyses, some associations with fat intake were found in men but were not replicated in women. Likewise, in men, the FTO polymorphism was associated $(\mathrm{P}<0.01)$ with lower alcohol intake but not in women. These associations, as well as others found with specific foods (legumes, dairy products, etc.), may be produced at random and require further replication.

Conclusion: Despite the association of FTO and MC4R gene variants with higher BMI, no relevant associations with food intake were found in this Mediterranean population. 
P5

Changes in Neutrophil Function Induced by Oral Administration of Oleic and Linoleic Acids in Rats

H.G. Rodrigues ${ }^{1}$, M.A. Vinolo', J. Magdalon', H. Fujiwara', E. Hatanaka ${ }^{1,2}$, R. Curi ${ }^{1}$

${ }^{1}$ Department of Physiology and Biophysics, Institute of Biomedical Sciences, São Paulo University; ${ }^{2}$ Institute of Physical Activity and Sport Sciences, Cruzeiro do Sul

University; São Paulo, Brazil

Objective: To investigate the effects of oral administration of oleic (OL) and linoleic (L) acids on neutrophil function and inflammatory response in rats.

Methods: Various doses $(25,50$, and $100 \mu \mathrm{L})$ of these fatty acids were given by gavage during 10 days. After this period, fatty acid composition of neutrophils (HPLC), expression of L-selectin and $\beta 2$-integrin (FACs), and production of proinflammatory cytokines (IL-1 $\beta$ and CINC-2 $\alpha \beta$ ) (ELISA) were determined. ANOVA and Dunnett's posttest were used for statistical analysis $(p<0.05)$.

Results: No alteration was observed in the final body weight and in food, water, and calorie intakes. Both fatty acids were not toxic to neutrophils in the doses given. The contents of oleic and linoleic acids were increased (by $100 \%$ and $30 \%$, respectively) but did not alter total saturated and polyunsaturated fatty acids in neutrophils. OL and L acids increased neutrophil migration, and this response was associated with augmented expression of L-selectin (1.53-fold for OL and 1.78 -fold for $\mathrm{L}$ ). Expression of $\beta 2$-integrin was not altered. Both fatty acids raised the production of IL- $1 \beta$ (2.75-fold for OL and 2.88-fold for $\mathrm{L}$ ) and CINC- $2 \alpha \beta$ (3.83-fold for OL and 8.25-fold for $\mathrm{L}$ ) after 4 hours of culture and reduced the release of IL- $1 \beta$ (by $56 \%$ for both) and CINC-2 $\alpha \beta$ (by $67 \%$ for OL and by $49 \%$ for $\mathrm{L}$ ) after 18 hours.

Conclusion: Oral administration of oleic and linoleic acids was incorporated into neutrophil lipids and raised L-selectin expression and neutrophil migration to the inflamed site induced by injection of glycogen solution. Both fatty acids stimulated the production of proinflammatory cytokines during the initial phase of the inflammatory response and returned their concentrations to basal levels in the resolution phase of inflammation. So, oleic and linoleic acids given by gavage are efficient to accelerate the onset and resolution of the inflammatory process.

Financial Support: FAPESP, CNPq, and CAPES

\section{P6}

Oral Tributyrin Administration Reduced Inflammation In Vivo and Inhibited Ex Vivo Production of Proinflammatory Mediators by LPS-Stimulated Neutrophils

\author{
Marco A.R. Vinolo', Hosana G. Rodrigues ${ }^{1}$, \\ Elaine Hatanaka ${ }^{1,2}$, Fábio T. Sato ${ }^{1}$, Rui Curi ${ }^{1}$ \\ ${ }^{1}$ Department of Physiology and Biophysics, Institute of \\ Biomedical Sciences, São Paulo University; ${ }^{2}$ Institute of \\ Physical Activity and Sport Sciences, Cruzeiro do Sul \\ University, São Paulo, Brazil
}

Objective: Butyrate is a short-chain fatty acid produced in the intestinal fiber fermentation by anaerobic bacteria. More than just being an important energy source for intestinal epithelial cells, butyrate is also a modulator of leukocyte function. The aim of this study was to evaluate the effects of butyrate on inflammatory process induced by intraperitoneal (i.p.) administration of glycogen.

Methods: Male Wistar rats were treated by oral route with different doses $(0.5,1.0$, and $3.0 \mathrm{~g} / \mathrm{kg}$ body weight) of a prodrug of butyrate (tributyrin). One hour after tributyrin administration, animals were i.p. injected with glycogen solution (1\%). Four hours later, neutrophils were collected and counted, and the production of proinflammatory mediators (TNF- $\alpha$, IL- $1 \beta, \mathrm{CINC}-2 \alpha \beta$, and NO) was assayed by ELISA.

Results: Rats treated with tributyrin (all doses) presented reduced recruitment of neutrophils (by at least $25 \%$ ) to peritoneum compared with the control group (treated with water). Neutrophils obtained from animals treated with tributyrin $3 \mathrm{~g} / \mathrm{kg}$ body weight produced less TNF- $\alpha$, CINC- $2 \alpha \beta$, and NO $(55 \%, 80 \%$, and $29 \%$ in comparison to control, respectively) after stimulation with LPS. Neutrophils from animals treated with lower doses of tributiryn $(0.5$ and $1.0 \mathrm{~g} / \mathrm{kg}$ body weight) also presented a significant reduction of CINC- $2 \alpha \beta$ production in response to LPS (90\%).

Conclusion: Results herein reported indicate that tributyrin presents consistent anti-inflammatory effects.

Financial Support: FAPESP, CNPq, and CAPES 


\section{P7 \\ Effect of Fatty Acids on Insulin Response, Mitochondrial Function, and Gene Expression in Skeletal Muscle Cells}

Sandro M. Hirabara ${ }^{1,2}$, Rafael H. Lambertucci ${ }^{1,2}$, Renato T. Nachbar', Luiz F.T. Camargo',

Leonardo R. Silveira ${ }^{3}$, Pierre Maechlert, Rui Curi'

${ }^{1}$ Postgraduate Program in Human Movement Sciences, Institute of Physical Activity and Sport Sciences, Cruzeiro do Sul University, São Paulo, Brazil; ${ }^{2}$ Department of Physiology and Biophysics, Institute of Biomedical Sciences, University of São Paulo, São Paulo, Brazil; ${ }^{3}$ Physical Education and Sports School, University of São Paulo, São Paulo, Brazil; ${ }^{4}$ Department of Cell Physiology and Metabolism, University Medical Centre, University of Geneva, Switzerland

Objectives: Increased plasma levels of free fatty acids (FFA) occur in states of insulin resistance such as obesity and type 2 diabetes mellitus. The high levels of plasma FFA are proposed to play an important role for the development of insulin resistance, but the mechanisms involved are still unclear. The effects of saturated and unsaturated FFA on insulin sensitivity and mitochondrial function in skeletal muscle cells were investigated. The effect of saturated FFA on production of reactive oxygen species (ROS) and the expression of citrate synthase and PPARgama coactivator-alpha (PGC-alpha) also was evaluated.

Methods: $\mathrm{C} 2 \mathrm{C} 12$ myotube cells were treated for $24 \mathrm{~h}$ with 0.1 $\mathrm{mM}$ of saturated (palmitic and stearic) or unsaturated (oleic, linoleic, eicosapentaenoic, and docosahexaenoic) FFA. After this period, basal and insulin-stimulated glucose metabolism and mitochondrial function were examined.

Results: Saturated palmitic and stearic acids decreased insulininduced glycogen synthesis by $22 \%$ and $18 \%$, respectively $(\mathrm{p}<0.01)$; glucose oxidation by $36 \%$ and $34 \%$, respectively $(\mathrm{p}<0.001)$; and lactate production by $32 \%$ and $25 \%$, respectively $(\mathrm{p}<0.001)$. Basal glucose oxidation was also reduced by $44 \%$ and $30 \%$, respectively $(\mathrm{p}<0.01)$. Palmitic and stearic acids impaired mitochondrial function as demonstrated by decreases in both mitochondrial polarization by $62 \%$ and $48 \%$, respectively $(\mathrm{p}<0.001)$, and ATP generation by $42 \%$ and $22 \%$, respectively $(\mathrm{p}<0.01)$. Production of ROS was concomitantly increased by these fatty acids: $35 \%$ for palmitic acid and $38 \%$ for stearic acid $(\mathrm{p}<0.01)$. There was no alteration in citrate synthase and PGC-alpha gene expression. In opposition to saturated FFA, unsaturated FFA did not impair glucose metabolism and mitochondrial function. Primary cultures of rat skeletal muscle cells exhibited similar responses to saturated FFA as compared to $\mathrm{C} 2 \mathrm{C} 12$ cells.

Conclusions: These results suggest that the impaired mitochondrial function and increased ROS production induced by saturated palmitic and stearic acids could be an early event in the development of insulin resistance in skeletal muscle cells.

Financial Support: FAPESP, CNPq, and CAPES
P8

\section{ALA16VAL SOD2 Polymorphism and Hypercholesterolemia on Lipids and Inflammation Biomarkers}

\author{
Ivana B.M. Da Cruz', Marta F.F. Duarte1, \\ Maria Gabriela Gottlieb², Rafael Noal Moresco3, \\ Thiago Duarte ${ }^{4}$, Maria Rosa Chitolina Schetingera ${ }^{5}$, \\ João Batista Teixeira Rocha ${ }^{5}$ \\ ${ }^{1}$ Universidade Federal de Santa Maria; ${ }^{2}$ Pontificia \\ Universidade Católica do Rio Grande do Sul; ${ }^{3}$ Centro de \\ Ciências da Saúde, Departamento de Farmacia; \\ ${ }^{4}$ Departamento de Farmacia, Vania Lucia Loroa, \\ Departamento de Química; ${ }^{5}$ Departamento de Quimica; \\ Maria Izabel de Ugalde Marques da Rocha, Departamento \\ de Morfologia, Universidade Federal de Santa Maria, \\ Santa Maria, Brazil
}

Introduction: Hypercholesterolemia-induced endothelial dysfunction due to excessive production of reactive oxygen species is a major trigger of atherogenesis. A previous study performed by our group found interaction between the polymorphism and hypercholesterolemia in levels of plasma lipid and inflammatory biomarkers using hypercholesterolemic subjects who were recently diagnosed and not yet treated and an association between ALA16VAL SOD2 manganese-dependent superoxide dismutase gene polymorphism and obesity.

Objective: Since the dismutation of superoxide is catalyzed by superoxide dismutase enzymes, we tested the association between hypercholesterolemia and ALA16VAL SOD2 polymorphism.

Methods: A case-control was performed including 915 subjects. We found a positive association between ALA16VAL SOD2 genotypes and hypercholesterolemia since lower A frequency was observed in the hypercholesterolemic group $(\mathrm{p}=0.001)$. The total cholesterol, LDL-chol, hs-CRP, OxLDL, and OxLDL autoantibody levels were not influenced by SOD2 polymorphism, just by hypercholesterolemia. However, hypercholesterolemic AV/VV genotype carriers showed significantly lower HDL cholesterol values compared to other groups.

Conclusion: A possible biological explanation for $\mathrm{VV}$ and hypercholesterolemia association could be a chronic state of superoxide imbalance present in VV carriers, which could affect differential metabolic pathways, triggering a lipid imbalance. Additional investigation to tested nutrigenetic effects need to be performed. 


\section{P9 \\ Interaction Effect of the Fatty Acid Synthase (FASN) Gene Polymorphisms and Lipid Intake on LDL Particle Size}

\author{
G. Dolley ${ }^{1,2,3}$, B. Lamarche ${ }^{3}$, J.P. Després ${ }^{4,5}$, C. Bouchard ${ }^{6}$, \\ L. Pérusse $e^{1,3,5}$, M.C. Voh/1,2,3 \\ ${ }^{1}$ Department of Endocrinology and Genomics, Centre \\ Hospitalier de l'Université de Montréal Research Centre, \\ Montréal, Quebec; ${ }^{2}$ Department of Food Science and \\ Nutrition; ${ }^{3}$ Institute of Nutraceuticals and Functional \\ Foods, Laval University; ${ }^{4}$ Quebec Heart Institute, Laval \\ Hospital, Saint-Foy, Quebec; ${ }^{5}$ Department of Social and \\ Preventive Medicine, Laval University, Québec City, \\ Quebec, Canada; ${ }^{6}$ Pennington Biomedical Research \\ Center, Baton Rouge, La., USA
}

The small, dense LDL phenotype is associated with an increased cardiovascular disease risk. A genome-wide scan performed on 236 nuclear families of the Quebec Family Study (QFS) revealed a quantitative trait locus (QTL) for LDL peak particle size (LDL-PPD) on the $17 \mathrm{q} 21$ region. A positional candidate gene was found in this region, the fatty acid synthase gene (FASN). FASN gene encodes a key enzyme involved in the biogenesis of membrane lipids. FASN may play a role in the regulation of feeding and may be a potential therapeutic target for obesity.

Objectives: To test the interaction between FASN gene polymorphisms and total lipid intake on LDL-PPD.

Methods: Analyses were performed on 592 subjects of QFS. Dietary fat was estimated by a 3-day food record. LDL-PPD was measured by gradient gel electrophoresis on nondenaturating $2-16 \%$ polyacrylamide gradient gels.

Results: Five single nucleotide polymorphisms (SNPs) were genotyped in FASN gene. None of the FASN SNPs were independently associated with LDL-PPD or with fat intake. However, FASN rs4246444 was associated with LDL-PPD in interaction with lipid intake $(p=0.001)$. Higher versus lower lipid consumers were also determined using a cutoff of $89 \mathrm{~g} /$ day, corresponding to the QFS median. Carriers of the variant allele showed smaller LDL-PPD only when consuming higher lipid amounts. This association remained significant after adjustments for age, sex, BMI, and triglyceride levels.

Conclusion: These results suggest that FASN gene influences LDL-PPD only in interaction with total dietary fat.

\section{P10}

\section{Genetic Factors Influencing Inflammation in Obesity}

Geneviève Faucher ${ }^{1,2,3}$, Véronique Garneau ${ }^{1,2,3}$, Luigi Bouchard 1,5, André Tchernof 1,3, Jean Bergeron ${ }^{1,6}$, Yves Deshaies 7 , Stéfane Lebel', Frédéric-Simon Hould ${ }^{8}$, Picard Marceau ${ }^{8}$, Marie-Claude Voh/1,2,3

'Department of Endocrinology and Genomics;

${ }^{2}$ Nutraceuticals and Functional Foods Institute;

${ }^{3}$ Department of Food Science and Nutrition, Laval

University, Quebec City, Quebec; ${ }^{4}$ Department of

Medicine, University of Montreal, Montreal, Quebec;

${ }^{5}$ ECOGENE-21 Research Center, Centre Hospitalier Affilié

Universitaire Régional, CSSS de Chicoutimi, Quebec;

${ }^{6}$ Faculty of Medicine, Laval University, Quebec City,

Quebec, Canada; ${ }^{7}$ Department of Anatomy and

Physiology and Laval Hospital Research Center, Quebec

City, Quebec; ${ }^{8}$ Department of Surgery, Medicine Faculty,

Laval University, Quebec City, Quebec, Canada

Obese individuals are often characterized by a chronic, low-grade inflammatory state. Previously, our group has shown that genes encoding for proteins involved in the anti-inflammatory and immune responses are differentially expressed in omental adipose tissue of obese men with or without the metabolic syndrome (MetS+/MetS-). Among these genes, the interferon-gamma-inducible protein 30 (IFI30) and thymic stromal lymphopoietin (TSLP) were selected for further genetic analyses.

Objective: The aim of the study was to verify whether IFI30 and TSLP gene polymorphisms contribute in explaining the well-known interindividual variability of the metabolic (MetS+/MetS-) and the inflammatory (low/high C-reactive protein (CRP) profiles observed in obesity.

Methods: We genotyped 1322 obese individuals for single nucleotide polymorphisms (SNPs) covering most of the sequence-derived genetic variability at the IFI30 and TSLP gene loci (5 SNPs for each gene). Genotype frequencies were tested in MetS+ vs. MetS- subgroups, defined according to the International Diabetes Federation Worldwide Definition of the Metabolic Syndrome, and in low- vs. high-plasma CRP subgroups defined using the median CRP value as a cutoff point.

Results: In the IFI30 gene, rs11554159 and rs7125 showed a significant difference in genotype frequencies between subgroups of low vs. high plasma CRP levels $(\mathrm{p}<0.05)$. Additionally, rs2289277 of the TSLP gene revealed significant differences in genotype frequencies between MetS+ vs. MetS- subgroups $(\mathrm{p}<0.05)$.

Conclusions: IFI30 and TSLP gene polymorphisms could partially explain the interindividual variability observed in the obesityassociated metabolic and inflammation alterations. 


\section{P11}

\section{ABCG5 Polymorphism Contributes to the Individual Response to Dietary Cholesterol and to Carotenoids Present in Eggs}

\author{
Maria-Luz Fernandez', Kristi Herron', José Ordovas², \\ Richard M. Clark, Ingrid E. Lofgren ${ }^{3}$ \\ ${ }^{1}$ University of Connecticut, Storrs, Conn.; ${ }^{2}$ Tufts University, \\ Boston, MA; ${ }^{3}$ University of Rhode Island, Kingston, R.I., \\ USA
}

The ATP binding cassette G5 (ABCG5) polymorphisms have been postulated to play a role in the response to dietary cholesterol. The objective of this study was to examine the contribution of the ABCG5 polymorphism on the plasma response to consumption of cholesterol and carotenoids from eggs. For this purpose, genotyping was conducted for 40 men and 51 premenopausal women who were randomly assigned to consume an egg (EGG, $640 \mathrm{mg} / \mathrm{d}$ additional dietary cholesterol and $600 \mu \mathrm{g}$ lutein+zeaxanthin) or placebo (SUB, $0 \mathrm{mg} / \mathrm{d}$ cholesterol, $0 \mu \mathrm{g}$ lutein+zeaxanthin) diet for 30 days. The two arms of the dietary intervention were separated by a 3 -week washout period. Plasma concentrations of total cholesterol, LDL cholesterol, and HDL cholesterol were determined. Because eggs are an excellent source of lutein and zeaxanthin, their plasma levels were also measured in a subset of subjects to determine whether the response to carotenoid intake was similar to that seen for dietary cholesterol and to evaluate the contribution of ABCG5 polymorphism to both responses. Individuals possessing the $\mathrm{C} / \mathrm{C}$ genotype experienced a greater increase in both LDL cholesterol $(\mathrm{P}<0.05)$ and a trend for lutein $(\mathrm{P}=0.08)$ during the EGG period when compared to those individuals with the $\mathrm{C} / \mathrm{G}$ (heterozygote) or $\mathrm{G} / \mathrm{G}$ genotypes (homozygotes). These results, although obtained from a small number of subjects, suggest that the ABCG5 polymorphism may play a role in the plasma response to dietary cholesterol and carotenoids.

\section{P12 \\ Zinc Deficiency Induces Upregulation of S100A8/A9 and Abolishes the Anticancer Effects of COX-2 Disruption in Murine Oral-Esophageal Cancer Prevention}

\author{
Louise Y. Fong', Shao-Gui Wan', Cristian Taccioli², \\ Yubao Jiang ${ }^{1}$, Xiu-Ping Liu'², Carlo M. Croce ${ }^{2}$, \\ John L. Farber ${ }^{1}$ \\ ${ }^{1}$ Thomas Jefferson University, Philadelphia, Pa.; ${ }^{2}$ The Ohio \\ State University, Columbus, Ohio, USA
}

Objectives: Zinc deficiency (ZD) is implicated in the pathogenesis of human oral-esophageal cancers. In addition, nutritional status affects the outcome of targeted cancer therapies. We tested the hypotheses that (1) with dietary ZD, cyclooxygenase-2 (COX-2) deletion enhances, rather than protects against, murine lingual carcinogenesis by 4-nitroquinoline 1-oxide (NQO) and that (2) under ZD conditions, alternative cancer pathway(s) are induced that abolish the beneficial effects of COX-2 pathway blockade.
Methods: Weanling $C O X-2-/-, C O X-2+/-$, and $C O X-2+/+$ (WT) mice were fed a ZD or $\mathrm{Zn}$-sufficient (ZS) diet to form 6 groups: ZD:COX-2-/-, ZD:COX-2+/-, ZD:WT, ZS:COX-2-/-, ZS:COX-2+/-, and ZS:WT. After 4 weeks, 14 ZD:COX-2-/-, 46 ZD:COX-2+/-, 19 ZD:WT, 14 ZS:COX-2-/-, 37 ZS:COX-2+/-, and 25 ZS:WT were given NQO in deionized water for 26 weeks for lingual tumor induction. Expression analysis was performed on forestomach mucosa from ZD:COX-2-/-, ZS:COX-2-/-, ZD:Wt, and ZS:Wt mice $(\mathrm{n}=4 /$ group) using GeneChip ${ }^{\circledR}$ Mouse Genome 4302.0 Array.

Results: With ZD, COX-2 disruption did not offer protection but caused high incidences of tumors (dysplasia/papillomas)/carcinomas in tongue/esophagus/forestomach in ZD:COX-2+/- and ZD:COX-2-/mice. Transcriptome analysis identified a gene signature in $\mathrm{ZD}: C O X-2-/-$ versus $\mathrm{ZS}: C O X-2-/-$ forestomach with overexpression of the proinflammation mediators $\$ 100 a 8 / a 9$, which were also overexpressed in $\mathrm{ZD}: \mathrm{COX}-2-/-$ tongue. Ingenuity Pathway Analysis uncovered an NF-kB centric network. Immunohistochemistry showed concomitant co-overexpression of S100A8/A9, RAGE (S100A8/A9 receptor), $\mathrm{NF}-\mathrm{kB}$ p65, cyclin $\mathrm{D} 1$, and tumor suppressor $\mathrm{p} 53$ protein in precancerous forestomach and carcinomas of tongue/esophagus/ forestomach, indicating that an inflammatory pathway (S100A8/ $\mathrm{NF}-\mathrm{kB}$ signaling) is activated under conditions of $\mathrm{ZD}$ and $\mathrm{COX}-2$ deletion. Zinc replenishment reduced inflammatory responses and hyperplasia in precancerous ZD:COX-2-/- forestomach.

Conclusions: The data provide proof of principle that $\mathrm{ZD}$ induces cancer-associated inflammatory responses and abolishes the intended effects of $C O X-2$ pathway blockade. Thus, zinc replenishment clinically may provide a promising strategy for improved targeted therapies.

Supported by NIH grant CA118560

\section{P13 \\ Polymorphisms in GSTM1 and GSTT1, Coffee Consumption, and Cutaneous Melanoma Risk \\ C. Fortes ${ }^{1}$, S. Mastroeni ${ }^{1}$, F. Melchi ${ }^{1}$, P. Anzidei², L. Innocenzi ${ }^{1}$, Giovinazzo Raffaella' ${ }^{2}$, G.M. Antonelli ${ }^{1}$, P. Pasquini ${ }^{1}$ F. Venanzetti ${ }^{2}$ \\ ${ }^{1}$ Istituto Dermopatico dell'Immacolata, Istituto di Ricovero e Cara a Carattere Scientifico; ${ }^{2}$ Technical Directorate for Risk Assessment and Prevention, Italian Workers' Compensation Authority; Rome, Italy}

Background: The glutathione S-transferase gene (GSTM1) has been reported to influence UV sensitivity and melanoma risk. There is increasing evidence identifying the role of dietary components in modulating the risk of melanoma. The aim of this study was to investigate GSTM1, GSTT1, coffee consumption, and the risk of cutaneous melanoma.

Methods: Within a case-control study aimed to study environmental and occupational risk factors for melanoma conducted in the inpatient wards of IDI-San Carlo Rome (Fortes et al. 2007), individual patterns at two polymorphic genes (GSTM1 and GSTT1) belonging to the glutathione S-transferase (GST) family were investigated in 340 subjects (188 cases of melanoma and 152 controls). The genetic 
analyses were conducted by INAIL. Information on sociodemographic characteristics, diet, smoking history, sun exposure, and pigmentary characteristics was collected for all subjects. The association between genetic polymorphyms, coffee consumption, and cutaneous melanoma was assessed by logistic regression.

Results: High frequency of coffee drinking ( $\geq$ once daily), compared with low-frequency consumption of coffee ( $<7$ times weekly), was associated with a protective effect for cutaneous melanoma (OR: 0.43; 95\% CI: 0.25-0.73) after controlling for age, sex, education, number of nevi, pigmentary characteristics, tobacco smoking, sun exposure, and polymorphic genes. After controlling for other food items simultaneously in the model, the protective effect of coffee remained. After stratifying for GSTM1 and GSTT1 genotypes, the protective effect was more pronounced for GSTM1 null genotype (OR: 0.35; 95\% CI: 0.15-0.78) and GSTT1 null genotype (OR: 0.031; 95\% CI: 0.006-0.30).

Conclusions: Our results show that consumption of coffee is protective for melanoma and that GSTM1 and GSTT1 null individuals may benefit more from this protection than GSTM1 and GSTT1 positive individuals.

Reference: Fortes C, Mastroeni S, Melchi F, Pilla MA, Alotto M, Antonelli G, Camaione D, Bolli S, Luchetti E, Pasquini P. The association between residential pesticide use and cutaneous melanoma. Eur J Cancer 2007;43:1066-75.

\section{P14 \\ Caveolin Functions in Fat and Skeletal Muscle of Diet-Induced Obese Rats: Relations With Insulin-Signaling Cascade in Fasting and Fed Conditions

\author{
A. Gómez-Ruiz', F.l. Milagro², J. Campión², J.A. Martínez², \\ C. De Migue/ ${ }^{1}$ \\ ${ }^{1}$ Department of Biochemistry and Molecular Biology, \\ ${ }^{2}$ Department of Nutrition and Food Sciences, Physiology \\ and Toxicology, University of Navarra, Pamplona, Spain
}

Objectives: We have analyzed the effects of a high-fat cafeteria diet on the expression and activation of caveolin 1, 2, and 3 in adipocytes and stromal cells from visceral and subcutaneous locations, as well as in skeletal muscle. We have also related these changes with the expression and function of different insulin-signaling intermediates in the same cell types.

Methods: Male Wistar rats were fed a control or cafeteria diet for 72 days. The night before sacrifice, some animals were fasted, whereas others remained fed. After sacrifice, gastrocnemius muscle was excised, adipocytes and stromal cells were isolated from retroperitoneal and subcutaneous depots, and serum metabolites were determined.

Results: The animals fed the cafeteria diet developed obesity. Insulin resistance, hyperglycemia, and hyperleptinemia were more markedly observed in the fasting state. As a consequence of feeding, Glut4 and Cav3 expression were upregulated in muscle and RT adipocytes from fed animals independently of the diet. Also, Cav2 phosphorylation was increased in the fed state in adipocytes from both fat depots, RT and SC. Cav1, however, showed the opposite response, being activated in RT adipocytes by fasting. The effects of feeding on some of these proteins are dependent on the type of diet. Thus, fasting-induced Cav1 activation and feeding-induced Cav2 phosphorylation were more evidently observed in rats fed the cafeteria diet. Nevertheless, feeding-induced Glut4 upregulation and muscle IR phosphorylation were not observed in cafeteria-fed rats, suggesting that a long period of feeding on a high-fat diet damages insulin-signaling cascade.

Conclusions: Rats fed the cafeteria diet show hyperglycemia and hyperinsulinemia. The effects of fasting are more evident in control-fed than in cafeteria-fed rats. In fact, the normal response to feeding, as observed in control animals, is not observed in cafeteria-fed rats. Thus, feeding induces changes in control-fed rats concerning Cav1 phosphorylation in RT adipocytes, IR activation in muscle, and Glut4 expression in all tissues. These changes are not observed in the cafeteria-fed animals, suggesting that this kind of diet alters the functionality and/or expression of these proteins. Finally, regarding the fat location, the diet and fasting effects on caveolin activation are more marked in visceral than in subcutaneous depot, suggesting that the former is more sensitive to the deleterious effects of high-fat diets than the subcutaneous depot.

\section{P15 \\ Role of Ceramide in Lymphocyte Death Induced by Docosahexaenoic Acid \\ Renata Gorjäo1, Fernanda Velame1, Karina Hoshino², Rui Curi \\ ${ }^{1}$ Department of Pharmacy, UNIBAN; ${ }^{2}$ Department of \\ Physiology and Biophysics, Institute of Biomedical \\ Sciences, University of São Paulo, São Paulo, Brazil}

Objective: In this study, we evaluated if docosahexaenoic acid (DHA, C22:6 n-3) modulates expression of proteins associated to apoptosis or proliferation of human lymphocytes in the presence or absence of the inhibitor of ceramide de novo synthesis, fumonisin B.

Methods: The toxicity of DHA in the presence of fumonisin B1 (FB1) was determined by flow cytometry. The effect of these compounds on lymphocyte proliferation, evaluated by thymidine incorporation, was investigated. Afterward, expressions of the proapoptotic proteins p53, bad and bax, antiapoptotic bcl-2 and bcl-xL, and proproliferative cyclin E and c-myc were determined by realtime PCR.

Results: DHA decreased cell proliferation at 50 and $75 \mu \mathrm{M}$. This effect, however, was abolished in the presence of FB1. DHA promoted an increase of $30 \%$ in DNA fragmentation at $75 \mu \mathrm{M}$. In the presence of FB1, this value was similar to control (without treatment). DHA promoted an increase of 82,85 , and $90 \%$ in expression of the pro-apoptotic $\mathrm{p} 53$, bax and bad genes, respectively, and a decrease of 50,64 , and $71 \%$ in expression of the bcl-2, bcl-xL, and c-myc genes, respectively. In the presence of FB1, the increase in expression of $\mathrm{p} 53$ and bad induced by DHA was abolished, and the expression of bcl-2, bcl-xL, and cmyc returned to control levels.

Conclusion: The inhibitory effect of DHA on lymphocyte proliferation involves ceramide production. DHA promotes an increase of intracellular ceramide content through de novo synthesis, leading to an increase in expression of pro-apoptotic proteins. In the absence of this sphingolipid, DHA was not able to induce cell arrest.

Financial Support: FAPESP, CAPES, and CNPq 


\section{P16 \\ Personalized Online Nutrition Guidance}

\section{Martin Kohlmeier}

The University of North Carolina at Chapel Hill, Chapel Hill, N.C., USA

Background: Optimal nutrient intake can prevent disease, support recovery, and delay recurrence. However, the optimal amounts of critical nutrients differ between people due to inherited, acquired, and environmental factors. Both observational studies and long-term intervention trials link an ever-increasing number of polymorphic genes to divergent intake thresholds. While many gene-nutrient interactions are still in question, the practical barriers to implementing intake targets for multiple nutrients have received little attention. It is obvious that not even nutrition experts can readily make food choices that closely meet their own needs, since the number of variables is simply too large.

Methods: A fully configured draft version of an online program for personalized nutrition guidance has been developed. It is now available at http://www.nim.unc.edu/guidance/guidance.html. This program fully supports the anonymized integration of genetic data for setting individual intake targets, as outlined previously (Kohlmeier, $J$ Nutrigenet Nutrigenomics 2008;1:303). Briefly, clients or their health care providers use for submission of DNA samples a computer-generated user code without personal information. Based on the analyzed genetic data, the laboratory enters adjustment and weighing factors in the database record associated with the user code. The online program combines the genetically derived factors with age, gender, weight, exercise level, and other modifiers to estimate nutrient intake targets. The program next selects food combinations that match most closely the user's personal profile. The nutrient information for the food items in the combinations is based on the USDA National Nutrient Database SR 21, augmented by label information from specific foods. At the outset, standard meal sizes are assumed, which can then be adjusted to match personal preference. The user can then review the suggested food combinations to see whether any are acceptable. The program shows users which foods would make a good combination when used in addition to a particular meal or as replacements for less favored items. Additional search functions support the sifting by preference (cereal type, ready meal, and other characteristics). Favorite meal combinations can be saved or printed out. The user can also review nutrient information of individual foods and link directly to external recipe sites where available.

Results: The feasibility of automated meal selection was tested by comparing breakfast pairings for two hypothetical individuals with differing weight, height, exercise level, and genetic make-up. Their folate intake targets were set to 82 and 122 micrograms per day, respectively. The offered 20 best food combinations provided an average of 104.3 (standard deviation 18.8) and 127 (SD 15.8) micrograms

Conclusions: Early experience with the program provides proof of concept for personalized online nutrition guidance. Specifically, this provides the first demonstration that genetic information can be used to inform individual food choices free of risks associated with the release of raw genetic data. Since this type of program cannot release discernible genetic information and does not impose onerous diets, genetic counseling seems not needed.

\section{P17 \\ MGMT ILE143VAL Polymorphism, Dietary Factors, and the Risk of Breast, Colorectal, and Prostate Cancer in the European Prospective Investigation Into Cancer and Nutrition (EPIC)-Norfolk Study}

Yet Hua Loh'1, Panagiota N. Mitrou², Richard Bowman ${ }^{3}$, Angela Wood ${ }^{1}$, Hannah Jeffery ${ }^{2}$, Robert Luben ${ }^{1}$, KayTee Khaw', Sheila Rodwel/ ${ }^{1}$

${ }^{1}$ University of Cambridge, Cambridge, UK; ${ }^{2}$ World Cancer Research Fund International, London, UK; ${ }^{3}$ Medical Research Council Dunn Human Nutrition Unit, Cambridge, UK

Objective: $O^{6}$-methylguanine-DNA methyltransferase (MGMT) repairs DNA damage caused by alkylating agents, including N-nitroso compounds from diet. MGMT ILE143VAL polymorphism may lead to less DNA damage repair and increased cancer risk depending on the environmental exposures. We investigated interactions between dietary factors and the MGMT ILE143VAL polymorphism in relation to breast, colorectal, and prostate cancer risk.

Methods: There were 276/1498, 273/2984, and 312/1486 cases/ controls for the breast, colorectal, and prostate cancer studies, respectively, all nested within the EPIC-Norfolk Study, a prospective cohort of approximately 25,000 men and women ages 45-79 years. Baseline 7-day food diary data were collected for dietary assessment.

Results: The MGMT ILE143VAL polymorphism was not overall associated with breast, colorectal, and prostate cancer risk. There was a significant interaction between this polymorphism and intake of red and processed meat on colorectal cancer risk $\left(P_{\text {interaction }}=0.04\right)$, suggesting an increased risk among carriers of the variant genotype compared to the MGMT ILE143ILE common genotype. A lower colorectal cancer risk was seen with higher intake of vitamin $\mathrm{E}$ and carotene among the variant genotype group but not in the common genotype group $\left(P_{\text {interaction }}=0.009\right.$ and $\mathrm{P}=0.005$ for vitamin $\mathrm{E}$ and carotene, respectively). A higher prostate cancer risk was seen with higher alcohol intake among the variant genotype $(\mathrm{OR}=2.08,95 \% \mathrm{CI}=1.21$ $\left.3.57, P_{\text {interaction }}=0.0009\right)$ compared to the common genotype with lower alcohol intake.

Conclusions: In this UK population, the MGMT ILE143VAL polymorphism was not associated with breast, colorectal, and prostate cancer risk. There was evidence for this polymorphism playing a role in modulating the risk of prostate cancer in the presence of alcohol. For colorectal cancer, MGMT ILE143VAL polymorphism may confer increased or decreased risk depending on the dietary exposure. 
P18

\section{Association Between Interleukin-1 Beta Polymorphism (+3953), Obesity, and Dietary Patterns}

Maria Fernanda Manica-Cattani', Ivana Beatrice Manica da Cruz², Maria Izabel de Ugalde Marques da Rocha', Ivo Emilio de Cruz Jung ${ }^{2}$, Thais Doeler Algarve2, Aron Ferreira da Silveira', Euler Esteves Ribeiro ${ }^{3}$

${ }^{1}$ Programa de Pós-Graduação em Bioquímica Toxicológica; ${ }^{2}$ Departamento de Morfologia, Laboratório de Biogenômica, Universidade Federal de Santa Maria, Santa Maria, Brazil; ${ }^{3}$ UNATI, Universidade Federal do Estado do Amazonas, Manaus, Brazil

Introduction: It now appears that obesity is associated with a low-grade inflammation of white adipose tissue resulting from chronic activation of the innate immune system such as interleukin-1 beta (IL-1 $\beta$ ). Previous investigations described a positive association between IL-1 $\beta+3953(\mathrm{C}>\mathrm{T})$ gene polymorphism and obesity. However, it is necessary to analyze if these results occur in other populations, are influenced by sex and age, and present interaction of diet patterns

Objective: To analyze the occurrence of association between IL-1 $\beta+3953(\mathrm{C}>\mathrm{T})$ gene polymorphism and obesity and nutrient consumption.

Methods: We performed a case-control study using 880 Caucasian subjects from the Brazilian Aging Research Program (nonobese $=283$, overweight $=334$, obese $=263$ ). We analyzed the IL-1 $\beta$ $+3953 \mathrm{C}>\mathrm{T}$ polymorphism

Results: We observed higher T allele (CT and TT) frequency in nonobese than in overweight and obese groups. The odds ratio showed 1.340 (95\% CI: $1.119-1.605)$ times more chance of the obese group being $C C$ carriers compared to nonobese group independent of sex and age. Obese TT ingested higher calories and saturated fat that other groups.

Conclusion: This study corroborates the idea that the IL-1 $\beta$ system is linked to the development of obesity and suggests the possible interaction of this association to dietary patterns.

\section{P19}

\section{SEL1L, a Gene Implicated in the ERAD Pathway, Is Upregulated During Intestinal Epithelial Differentiation Along the Enterocytic and Paneth Lineages and Is Overexpressed in Differentiated Colorectal Cancer}

\author{
Hassan Ashktorab1, William Green ${ }^{1}$, Giovanna Finzi², \\ Fausto Sessa 2,3,4, Mehdi Nouraie1, Edward L. Lee1, \\ Annalisa Morgano6,7, Antonio Moschetta7, \\ Massimo Barberis ${ }^{8}$, Monica Cattaneo, \\ Lavinia Vittoria Lotti9, Ida Biunno ${ }^{5}$, Renato Mariani- \\ Costantini 6
}

${ }^{1}$ Department of Medicine and Cancer Center, Department of Pathology, Howard University, College of Medicine, Washington, D.C., USA; ${ }^{2}$ Department of Pathology, Ospedale di Circolo, Varese, Italy; ${ }^{3}$ Department of Human Morphology, University of Insubria, Varese, Italy; ${ }^{4}$ Department of Pathology, Multimedica IRCCS, Milan, Italy; ${ }^{5}$ Institute for Biomedical Technologies, National Research Council, Segrate, Milan, Italy; ${ }^{6}$ Department of Oncology and Neuroscience, Gabriele d'Annunzio University, and Center of Excellence on Aging, Gabriele d'Annunzio University Foundation, Chieti-Pescara, Italy; ${ }^{7}$ Laboratory of Lipid Metabolism and Cancer, Department of Translational, Pharmacology, Consorzio Mario Negri Sud, S. Maria Imbaro, Chieti, Italy; ${ }^{8}$ Ospedali RiunitiBergamo, ${ }^{9}$ Department of Experimental Medicine and Pathology, University of Rome, Rome, Italy

Objectives: We investigated the role of SEL1L, a gene of the endoplasmic reticulum (ER)-associated protein degradation (ERAD) pathway, in the differentiation of human intestinal epithelia and its possible involvement in colorectal cancer.

Methods: SEL1LA mRNA and protein expression were investigated in relation to biochemical and morphological markers of differentiation in the human colorectal cancer cell lines $\mathrm{CaCo} 2$ and HT29, which provide in vitro models of intestinal epithelial differentiation. Localization of SEL1L along the crypt to villus axis was investigated by immunohistochemistry and immunoelectron microscopy on biopsies of human duodenal mucosa. SEL1L protein expression was then analyzed by immunohistochemistry on tissue microarrays, including 134 colorectal adenomas and 58 colorectal carcinomas from African American patients and matched normal colorectal mucosa. Distribution for percentage of stained cells was studied by computing median and interquartile range due to skewed distribution. The nonparametric Kruskalwalis test was applied to verify differences in percentage of stained cells.

Results: Along the crypt to villus axis, SEL1L immunostaining strongly colocalized with lysozyme in Paneth cells (crypt bases). Less strong cytoplasmic expression was seen in differentiating enterocytic progenitor cells and mature enterocytes. In HT29 and $\mathrm{CaCo} 2$ cells, SEL1L mRNA and protein levels increased upon spontaneous and induced differentiation, together with CDX2, p21, and KLF4. Subcellular localizations showed colocalization with calreticulin and with markers of autophagy, including LC3, and varied in relation to glandular differentiation. In colorectal tumors, SEL1L expression correlated significantly with progression from adenoma to carcinoma 
$(\mathrm{P}=0.0001)$ and was stronger in well-differentiated to moderately differentiated compared to poorly differentiated colorectal cancers.

Conclusions: SEL1L analysis in the HT29 and $\mathrm{CaCo} 2$ cells and in vivo studies on normal intestinal mucosa indicate that SEL1L expression increases with cell differentiation, being particularly strong in Paneth cells, which actively synthesize secretory defensins and lysozyme. Well-differentiated colorectal carcinomas contain a high fraction of transformed cells that progress toward a differentiated secretory phenotype, which may explain SEL1L upregulation in these tumors. SEL1L expression could provide a biomarker that identifies colorectal cancer cell populations with differentiated secretory phenotype, acute ER stress, and active ERAD response.

Acknowledgments: This work was supported in part by Grants \#CA102681 and CA90890 funded by the National Cancer Institute, NIH; by the Italian Government MIUR-FIRB grant nRBIP064CRT; by Italian Government MIUR 60\% grants to RMC for 2008 and 2009; and by a grant from Associazione Italiana per la Ricerca sul Cancro to AM. Dr. A. Morgano is a Ph.D. student in the oncology program at Gabriele d'Annunzio University, Chieti, Italy.

\section{P20 \\ Breakthrough Database on Genetics and Health With Controlled Vocabulary and Drill- Down Discovery Tools}

\author{
Ron L. Martin \\ Nutrigenetics Unlimited, Inc., Fullerton, Calif., USA
}

Objectives: Extensive subset of PubMed references on the interplay between genetics, health, and our environment - including but not limited to nutrition. Intended to inform the public, equip professionals, and encourage public/professional partnerships (www.nutrigenetics.net).

Methods/Features: Controlled vocabulary loosely based on Medical Subject Heading (MeSH) terms. User-selectable indexes for any given topic or for combinations of several topics. Favorite topics list of up to 50 topics can be selected by each user. $275,000+$ references spanning the last 10 years: 2000-to date; 2,000+ relevant new articles added monthly; $1,000+$ health conditions; $1,000+$ risk modifiers (including $~ 300$ diet- or supplement-related) 1,000+ genes and genetic variants (more added daily and/or on request); $500+$ other topics, including $100+$ specific countries.

Results/Benefits: Controlled vocabulary solves the problem of inconsistent terminology. User-selectable indexes show the number of references for each subtopic, plus synonyms. Drill-down discovery occurs with stepwise use of user-selectable indexes. Any given subtopic can be expanded to reveal its own list of subtopics and then repeated again to reveal highly focused combinations of topics that would otherwise easily escape attention. Favorite topics list allows users to easily identify new references that are relevant to their own interests or to the interests of family, friends, or colleagues. Also identifies topics that may warrant enhanced coverage in response to high user interest.

Conclusions: Solving terminology inconsistencies, providing drill-down discovery tools, and facilitating updates on favorite topics - together constituting a breakthrough in revealing the hidden relationships between genetics and our environment. Informing the public, equipping professionals, and encouraging public/professional partnerships.

\section{P21 \\ PPAR-Alpha-Dependent Response to Plant Diets}

Kiyoto Motojima, Machiko Todokoro, Bunichiro Ashibe

Meiji Pharmaceutical University, Tokyo, Japan

Animals have developed the genomic system to detoxify or exclude potentially toxic compounds in food, most of which are produced by plants as secondary metabolites. The detoxification system should be tightly regulated with the nutrient absorption system, but studies on these mechanisms were limited because to date most animal experiments have been carried out using laboratory diets. Using plant seeds as a diet for mice, we found large genotype and genderdependent differences in response to the plant food by performing transcriptome and metabolome analyses.

Although mice lacking peroxisome-proliferator-activated receptor $\alpha(\operatorname{PPAR} \alpha)$ display few abnormalities in lipid metabolism, especially during youth, under standard laboratory conditions they responded quite differently to the natural diets when compared with wild-type mice. Feeding sesame seeds for a few days, as a most extreme example, caused a drastic fatty liver with unbalanced fatty acid composition in PPAR $\alpha$-null male mice. DNA microarray analysis of the sesame-induced mRNAs in the intestine and liver revealed that PPAR $\alpha$ plays a vital role in inducing various xenobiotic metabolizing enzymes (FEBS J 273, 292 2006). However, the changes in the expression levels of mRNAs for fatty acid remodeling enzymes were too small to explain the drastic change in the fatty acid composition in the fatty livers of the PPAR $\alpha$-null mice.

Among several changes evidently observed in the PPAR $\alpha$-null male mouse liver, we had an interest in the increases in the amount of $\gamma$-linolenic acid (C18:3) and octadecatrienoic acid (C18:4) and the concomitant decreases in the amount of dihomo- $\gamma$-linolenic acid (C20:3) and eicosatrienoic acid (C20:4) and analyzed the cause of the changes using the RNAi knocking down and stable isotope approaches in human hepatoma $\mathrm{HuH} 7$ cells. Sesame-induced changes in the fatty acid composition in the knockout male mouse liver could be explained not by transcriptional repression but by specific inhibition of the activity of very-long-chain fatty acid elongase 5 (Elovl5). Screening of the sesame lignans possibly involved in the disturbance revealed that matairesinol and arctigenin have inhibitory activities against the elongase 5.

These results together suggest that some lignans in sesame seeds cause the disturbance of fatty acid metabolism in the liver by inhibiting the elongase 5 if they are not metabolized to inactive compounds in a gender-specific and/or PPAR $\alpha$-dependent manner. We speculate that animals have developed the systems to detoxify plant secondary metabolites during establishment of the food chain, and dysregulation of the system could cause severe ill effects in animals. Understanding of these relationships should be an important area of nutrigenomics. 


\section{P22}

\section{Use of Folic Acid Among Pregnant Women With Congenital Anomalies in a Hospital Population}

Nuzhat Nauman', Samina Jalali², Sarwat Jehan², S.A.Shami², Riffat Shaheen ${ }^{3}$, Rehana Hamid ${ }^{3}$

${ }^{1}$ Rawalpindi Medical College, Rawalpindi, Pakistan; ${ }^{2}$ Quaid-i-Azam University, Islamabad, Pakistan; ${ }^{3}$ Federal Government Services Hospital, Islamabad, Pakistan

The prevalence of neural tube defects is high in Pakistan. Daily consumption of a supplement containing $400 \mathrm{ug}$ folic acid before and during the first trimester of pregnancy has been shown to be effective in preventing both the occurrence and recurrence of neural tube defects.

Methods: The study population comprised 70 pregnant women coming for delivery/termination to the Obstetrics Department, Federal Government Services Hospital, in Islamabad from January 2004 to June 2004.

Results: Among the congenital anomalies, the percentage of neural tube defects was $58.57 \%$, anencephaly $38.57 \%$, spina bifida $12.85 \%$, and meningocele $7.14 \%$. In the study sample, $10 \%$ of women took iron or multivitamin preparations. Dietary intake of food containing folic acid was inadequate. Folic acid awareness was $10 \%$, and knowledge regarding the role of folic acid in the prevention of neural tube defects was zero percent. In the study sample, $84 \%$ of patients were from urban areas, and $16 \%$ were from rural areas. The majority of patients were illiterate or were educated only up to the primary level and belonged to a lower socioeconomic class.

Conclusion: The levels of folic acid awareness and knowledge and use of folic acid are extremely low. Efforts, including public health campaigns aimed at increasing the awareness, knowledge, and periconceptional use of folic acid, are urgently needed, both in rural and urban areas, with special emphasis to the less educated, lower socioeconomic class.

\section{P23}

Maternal Saturated-Fat Diet During Lactation Improves Glucose Tolerance and Influences Gene Expression in Adult Mice
V. Palsdottir 1,2, B.G. Gabrielsson 2,4, A. Wickman³, N. Andersson ${ }^{3}$, B. Strandvik ${ }^{1}$
${ }^{1}$ Department of Paediatrics, Institute of Clinical Sciences, Sahlgrenska Academy; ${ }^{2}$ Department of Clinical and Molecular Medicine, Institute of Medicine, Sahlgrenska Academy; ${ }^{3}$ Department of Physiology, Institute of Neuroscience and Physiology, Sahlgrenska Academy, Gothenburg University, Gothenburg, Sweden; ${ }^{4}$ Department of Chemical and Biological Engineering, Food Science, Chalmers University of Technology, Gothenburg, Sweden

Objectives: Malnutrition in early life can program for altered metabolism in adult life, but the impact of fat quality in the maternal diet has not been thoroughly studied. Our aim was to investigate if a diet with saturated fat without essential fatty acids during the lactation period in mice could affect the short- and long-term metabolism and the hepatic gene expression of PPARs in the offspring.

Methods: Mice of C57BL/6 strain were given a diet containing saturated fat (SF, $\mathrm{n}=10)$ ) or a control diet with a mixed-fat profile $(n=10)$ from day 16 of pregnancy and throughout 3 weeks of lactation. One male pup from each litter $(n=10)$ was killed at weaning $(3$ weeks), and the liver was frozen until analysis of mRNA with realtime PCR. The remaining mice were weaned to ordinary chow, and at 15 weeks of age one group of former SF offspring and one group of controls were given high-fat diet. The body weight was measured weekly, and body composition measurements by DEXA and intraperitoneal glucose tolerance test were performed on the adult animals before they were killed at 21 weeks of age. The liver and blood were collected for mRNA analysis and leptin ELISA, respectively.

Results: The body weight and plasma leptin of SF pups were lower at 3 weeks of age compared to controls $(p>0.001)$ and remained so in both sexes during the whole study. The hepatic mRNA expressions of PPARg and its coactivator PGCla were increased $(p<0.05)$, but PPARa and PPARd were unchanged in the SF offspring compared to the controls at 3 weeks of age. The upregulation of PGC1a was persistent into adult age in the male SF mice, but PPARg was downregulated in the same mice compared to controls $(\mathrm{p}<0.05)$. The adult SF mice had lower fat mass compared to controls at both 15 and 20 weeks of age. The SF mice of both genders had improved glucose tolerance with lower insulin levels at 20 weeks of age compared to controls, and this fact was further potentiated after high-fat diet.

Conclusions: Our results show that fat quality in the diet during the early postnatal period is critical in determining adult body weight and glucose metabolism. Saturated-fat diet during lactation resulted in resistance against obesity and increased the insulin sensitivity, also after challenge with high-fat diet for 5 weeks. The effect was associated with persistent low leptin concentrations and changed gene expression in the liver.

P24

\section{The Genome-Wide Association Study of Metabolic Syndrome and Glycemic Load in Korea: Korean Genome and Epidemiology Study}

\section{S.J. Park, M.J. Go, Y. Ahn, S.S. Kim \\ Center for Genome Science, Centers for Disease Control and Prevention, Seoul, Korea}

Objectives: To identify susceptibility genes for metabolic syndrome (MS) through a genome-wide association study (GWAS) and to investigate the association of genotype and risk of MS by glycemic load (GL).

Methods: The GWAS tested $500 \mathrm{~K}$ single-nucleotide polymorphisms (SNPs) using Affymetrix chips and included 2,857 MS cases and 5,939 controls from a community-based cohort. GL was calculated from a food frequency questionnaire (103 food items).

Results: Twenty-one SNPs were significantly associated with MS by logistic regression analysis after adjusting age, sex, and area $\left(\mathrm{p}<10^{-5}\right)$. Four SNPs (rs154168, rs154167, rs10509208, rs6015068) 
showed significant association with GL value. The prevalence of MS was higher in the highest quintile GL group than in the lowest quintile group. In the lowest quintile GL group, MS risk is significantly higher among the risk allele carriers than noncarriers (OR: $2.0495 \% \mathrm{CI}$ : $1.16-3.58, \mathrm{p}=0.0129$ in rs 154167 , OR: $1.4395 \% \mathrm{CI}: 1.05-1.94$, $\mathrm{p}=0.025$ in rs10509208).

Conclusion: These results suggested that GL had positive association with MS prevalence, and some risk allele carriers showed higher risk for MS than nonrisk allele carriers even in the low GL group.

\section{P25 \\ Comparative Effect of Saturated and Polynsaturated Fatty Acids on Human Neutrophil Death}

\author{
A.C. Levada-Pires', T.M. Lima', E. Hatanaka², R. Curi', \\ T.C. Pithon-Curi ${ }^{2}$ \\ ${ }^{1}$ Department of Physiology and Biophysics, Institute of \\ Biomedical Sciences, University of São Paulo, São Paulo, \\ Brazil; ${ }^{2}$ Post-Graduate Program in Human Movement \\ Sciences, Institute of Physical Activity Sciences and \\ Sports, Cruzeiro do Sul University, São Paulo, Brazil
}

High concentration of certain fatty acids (FA), particularly polyunsaturated fatty acids (PUFA), can cause cell death via apoptosis or, when concentrations are even greater, necrosis. In the present study, the toxicity of oleic, linoleic, docosahexaenoic (DHA), stearic, and palmitic acids on human neutrophils was examined. Neutrophils from sedentary volunteers were cultivated for 3 hours with increasing concentrations of these fatty acids (from 50 to $300 \mu \mathrm{M}$ ). Cell membrane integrity, DNA fragmentation, and phosphatidylserine externalization were evaluated by flow cytometry. The mechanisms involved in the toxic effects of the FA on neutrophils were also investigated by measuring ROS production and mitochondrial transmembrane polarization. The maximal tolerable (nontoxic) concentration of the FA was $200 \mu \mathrm{M}$ for oleic, linoleic, DHA, and palmitic acids and $100 \mu \mathrm{M}$ for stearic acid. So, stearic acid was the most potent fatty acid tested to cause human lymphocyte death. Oleic, linoleic, DHA, and palmitic acids increased DNA fragmentation and phosphatidylserine externalization at toxic concentrations $(250 \mu \mathrm{M})$, suggesting the occurrence of neutrophil death by apoptosis. The percentage of cells with intact plasma membrane decreased due to addition of oleic acids (250 and $300 \mu \mathrm{M})$ and DHA $(300 \mu \mathrm{M})$. These latter observations indicate the occurrence of secondary necrosis. The effect of oleic, linoleic, DHA, and palmitic acids on neutrophil apoptosis was associated to an increase of ROS production and mitochondrial membrane depolarization. Mitochondrial pore formation leads to cytochrome c leakage and activation of caspases that induces degradation of target proteins involved in DNA fragmentation. So, oleic, linoleic, DHA, and palmitic acids induced apoptosis by change in mitochondrial function, suggesting that this process was induced via intrinsic pathway. Stearic acid induced DNA fragmentation with no change in phosphatidylserine externalization and mitochondrial transmembrane depolarization. These results indicate that stearic acid induced neutrophil death by other mechanism rather than the classical apoptotic pathway. In conclusion, except for stearic acid, the fatty acids tested induce human neutrophil death by apoptosis, and this toxic effect involves increased ROS production and mitochondrial membrane depolarization.

Financial Support: FAPESP, CAPES, and CNPq

\section{P26 \\ Influence of Omega-3 Fatty Acids and the PPAR-Alpha L162V Polymorphism on Cytokine Gene Expression Levels in Human Macrophages}

Iwona Rudkowska, Christophe Garenc, Patrick Couture,
Marie-Claude VohI

Lipid Research Center, Centre Hospitalier de I'Université

Research Center, and Nutraceuticals and Functional Foods Institute, Quebec City, Quebec, Canada

Omega-3 fatty acids (FAs) mediate anti-inflammatory effects through the activation of the peroxisome proliferator-activated receptor $\alpha$ (PPAR $\alpha)$, a transcription factor that regulates the expression of cytokine genes. Human studies have reported decreased production of tumor necrosis factor alpha (TNF $\alpha$ ), interleukin-6 (IL6), and interleukin-1 beta (IL1 $\beta$ ) after omega-3 FA supplementation, yet others demonstrated that omega-3 FA supplementation does not reduce proinflammatory cytokine release. The $\mathrm{L} 162 \mathrm{~V}$ polymorphism of the PPAR $\alpha$ gene has been associated with a deteriorated metabolic profile in numerous studies and thus may influence individuals' antiinflammatory response.

Objective: To examine whether subjects carrying the V162 allele exhibit differences in the expression levels of these cytokines in the presence of omega-3 FAs compared to L162 homozygotes.

Methods: Peripheral blood monocytes from six men carrying the $V 162$ allele paired for age and for body mass index with six L162 homozygotes were differentiated into macrophages and incubated with $10 \mu \mathrm{M}$ eicosapentaenoic acid (EPA), $10 \mu \mathrm{M}$ docosahexaenoic acid (DHA), or mixtures of 10:5 $\mu \mathrm{M}$ and 5:10 $\mu \mathrm{M}$ EPA: DHA for 48 hours. Afterward, mRNA expression levels of PPAR $\alpha, T N F \alpha, I L 6$, and $I L 1 \beta$ were quantified by real-time polymerase chain reaction (RT-PCR).

Results: We found that gene expression levels of PPAR $\alpha$ were significantly lower for carriers of the V162 allele compared to L162 homozygotes after the addition of $10 \mu \mathrm{M}$ DHA and a mixture of 10:5 $\mu \mathrm{M}$ EPA:DHA. Yet, TNF $\alpha$, IL6, and IL1 $\beta$ gene expression rates did not display differences between the subjects with or without the $L 162 \mathrm{~V}$ polymorphism after the addition of omega-3 FAs.

Conclusion: These results suggest that subjects bearing the $P P A R \alpha L 162 \mathrm{~V}$ polymorphism exhibit a lower expression of PPAR $\alpha$; however, this particular polymorphism alone does not significantly influence the anti-inflammatory response to omega-3 FA supplementation.

Funding provided by a CIHR Operating Grant 


\section{P27}

\section{Silencing of the RARA Epigenetic Network Reveals the Tumor-Promoting Activity of Vitamin A}

\author{
Stefano Rossetti, Ming Qiang Ren, Giulia Somenzi, \\ Luigina Tagliavacca, Nicoletta Sacchi \\ Cancer Genetics Program, Roswell Park Cancer Institute, \\ Buffalo, N.Y., USA
}

Objectives: Preformed vitamin A, or retinol, is found in animal foods such as liver and whole milk and in some fortified food products. Carotenoids such as beta-carotene from fruit and vegetables are also efficiently converted into retinol. Retinol converted into its bioactive form retinoic acid (RA) plays a major role in cell division, cell death, and cell differentiation. Work from our laboratory has shown that RA is a double-edged molecule: It can either inhibit or promote cell tumorigenesis. We traced the paradoxical tumor-promoter activity of RA to the functional activity of RA receptor alpha (RARA). Lack or functional inhibition of RARA apparently leads to concerted epigenetic silencing of a network of tumor suppressor genes controlling cell death (nSMase), cell proliferation (RARB2), and migration and invasion (TGFBR2). In addition, RA, likely through noncanonical RAR targets, activates prosurvival and prometastatic signaling pathways. The aim of this study was to use preclinical xenograft mouse models to test whether dietary RA and the RA precursor retinol can promote the in vivo growth of cancer cells that, due to functional inhibition of RARA, have developed an epigenetically silent tumor-suppressor gene network.

Methods: An isogenic model of breast cancer (T47D), comprising two clonal cell lines with $\left(\mathrm{T} 47 \mathrm{D}^{\mathrm{LXC}}\right)$ and without $\left(\mathrm{T} 47 \mathrm{D}^{\mathrm{DNC}}\right)$ functional RARA (Ren et al., $M C B, 2006$ ), was used to generate subcutaneous xenograft tumors in athymic female nude mice. We analyzed in two independent experiments the growth of T47D $\mathrm{D}^{\mathrm{LX} 5}$ and T47D ${ }^{\text {DNC8 }}$ xenograft tumors of mice (10 mice/group) fed a diet (1) free of RA and retinol, (2) with RA (5 or $10 \mathrm{mg} / \mathrm{kg}$ ), and (3) with retinol $(50 \mathrm{mg} / \mathrm{kg})$ for up to 6 weeks. Xenograft tumor volume was measured twice a week; at the end of the sixth week, tumors were excised and analyzed for histological and molecular markers of cell proliferation. Data were analyzed by one-way ANOVA, followed with multiple comparison tests. All statistical tests were two sided. The level of significance was set at $\mathrm{p}<0.05$.

Results: Both dietary RA and dietary retinol significantly promoted the growth of $\mathrm{T} 47 \mathrm{D}^{\mathrm{DNC} 8}$ xenograft tumors and significantly inhibited the growth of T47D $\mathrm{D}^{\mathrm{LXC} 5}$ xenograft tumors.

Conclusions: Breast cancer cells with a functionally impaired RARA leading to an epigenetically silent tumor-suppressor gene network are susceptible of being growth-promoted by dietary RA and its precursor retinol. The identification of genetic/environmental factors that can induce RARA functional inactivity should help prevent the epigenetic silencing of the RARA-regulated tumor-suppressor gene network. Early detection of tumors with RARA functional inactivity (e.g., evidence of epigenetic silencing of tumor-suppressor genes of the RARA network) may limit the potential danger of dietary vitamin A on breast tumorigenesis.

This work was supported in part by R01 CA127614 to NS; SR was the recipient of a Susan Komen postdoctoral fellowship; GS was supported by the Graduate Program of Molecular Medicine, Medical School, University of Milan; and LT was supported by Cariplo Foundation 2006-0788 grant.

\section{P28 \\ The Nutritional Hypothesis of Women's Higher Health Risk With a High N-6 PUFA DIET vs. Men's Better International Standing: A Gender "Paradox"?}

Niva Shapira

Stanley Steyer School of Health Professions, Tel Aviv University, Tel Aviv, Israel

Objectives: Suggested links between high n-6 PUFA consumption and unexpectedly low international health rankings among Israelis despite an otherwise "good" diet, with women's worse vs. men's better standing, have raised a gender dichotomy hypothesis of n-6 PUFA-differentiated risk.

Methods: International and Israeli health surveys and national registries were searched and compared for disease incidence, mortality rates, and dietary patterns.

Results: Israeli health status was found to be lower than in most other Mediterranean countries, despite the "good" Israeli diet-low in calories and fat and high in PUFA:SFA ratio, fruits, and vegetables. This unexpected dissociation was defined as the "Israeli paradox" and was attributed to high consumption of n-6 PUFA. However, gender analysis showed that only women ranked unexpectedly low (11/15 European countries in life expectancy), whereas men ranked higher than most. This could suggest a "gender dichotomy" of differential health profiles with the same diet, which appears to be concentrated in cancer risk. Israeli women rank worse than men for all-cancer mortality (15 vs. 37 among 44 European countries); mortality from breast cancer among Israeli women is $19 \%$ higher than Eur-A, while prostate cancer among Israeli men is $25 \%$ lower. Nationally, breast cancer has contributed $>200 \%$ more to women's mortality than prostate cancer has to men's $(21.4 \%$ vs. $9.8 \%)$ and to national mortality ( $17.1 \%$ vs. $8.2 \%)$.

Trends of gender-specific risk were also observed in comparisons of ethnic subgroups. Jewish women have had much higher cancer prevalence (1:3) than Israeli-Arab women (1:6) and 1.7 times higher cancer mortality with higher PUFA:MUFA ratio (1.7:1 vs. 1.2:1). However, Israeli-Arab women now have a significantly faster growing rate (by 5.3 vs. $-3.3 \%, 1980-2000$ ), whereas men have remained relatively stable. Of note, Israeli-Arab women have significantly higher obesity rates and slightly higher all-heart disease and diabetes mortality rates, with smaller annual decreases compared to Jews. The general decline observed in Arab health status, especially in women, is concurrent with dietary westernization, including exchanging the traditional olive oil (high in n-9 MUFA) for high n-6 PUFA oils (mostly soy), resulting in increasing $n-6$ consumption and high dietary n-6:n-9 and n-6:n-3 FA ratios. Some research has demonstrated hormonal and sexual dimorphism in n-6 PUFA metabolism and enhanced carcinogenicity that may be adjunctive to genetic predisposition, as well as protective effects of olive oil against breast cancer mutations.

Conclusions: This is the first time sexual dimorphism has been suggested as a modulating factor of dietary effects on public health, linking dietary westernization and n-6 PUFA consumption to western health risks. Supporting evidence may suggest that gender and/or genetic predisposition not only does not preclude the role of nutritionbased prevention but also may increase its importance. In view of the global increase in n-6 PUFA consumption, Israeli women may represent a case study for research into gender-based nutritional intervention, especially as related to cancer epidemiology. 


\section{P29}

\section{Changes in Proinflammatory Cytokine Expression by Fatty Acids in Endothelial Cells}

\author{
Érica Paula Portioli Silva', Laureane Nunes Masi', \\ Thais Martins de Lima Salgado², Rui Curi ${ }^{1}$ \\ ${ }^{1}$ Institute of Biomedical Science, Department of \\ Physiology and Biophysics; ${ }^{2}$ Department of Medical \\ Clinic, Medicine School; University of São Paulo, São \\ Paulo, Brazil
}

Activation or dysfunction of the vascular endothelium is one of the first events in the development of inflammation and atherosclerosis. Inflammatory reactions involve chemokines, inflammatory cytokines, and adhesion molecules, whose expression is regulated by a variety of transcription factors, including nuclear factor $\kappa \mathrm{B}(\mathrm{NF}-\kappa \mathrm{B})$. Fatty acids (FA) can activate NF- $\mathrm{B}$ in endothelial cells. The information above led us to investigate the effect of various FA [palmitic PA- (16:0), stearic - SA- (18:0), oleic - OA - (C18:1), linoleic - LA (18:2), eicosapentaenoic - EPA - (20:5), and docosahexaenoic - DHA - (22:6) acids] on expression of interleukin IL-1 $\beta$, IL-6, IL-8, and ICAM-1 and activation of NF- $\mathrm{KB}$ in ECV-304 endothelial cell line in culture. All FA induced an increase in expression of IL-1 $\beta$ (by approximately three times) compared to vehicle (ethanol), whereas expression of IL-6 gene genes was increased by saturated FA (SFA) and polyunsaturated FA (PUFA), and IL-8 gene expression was increased by PUFA. PA and SA raised IL-6 expression by 6 -fold, and 10 -fold, respectively. DHA provoked an increase of IL-6 expression by 8 -fold and EPA augmented it by 60 -fold. The IL- 8 gene expression was increased (by 7-fold) by DHA and EPA. Activation of NF-кB was markedly stimulated by PA, SA, EPA, and DHA after 30 minutes of treatment. These findings indicate that SFA and PUFA raise the expression of proinflammatory genes in endothelial cells, probably due to activation of NF-кB. These observations provide important information for the development of new therapeutic strategies to prevent atherosclerosis.

This research is supported by FAPESP, CNPq, and CAPES.

\section{P30 \\ Antioxidant Properties of Wild Plants Used as Food in the Mediterranean Basin}

\author{
Linda Sacchetti, Paola Vanzani, Monica Rossetto, \\ Veronica De Marco, Tiziano Gomiero, Adelio Rigo, \\ Maurizio G. Paoletti \\ University of Padua, Padua, Italy
}

The Mediterranean diet, which is rich in plant foods, appears to afford protection against degenerative diseases such as cardiovascular and neurological disorders. Research on the properties of "minor components" of the wild herbs used in the Mediterranean diet (e.g., polyphenols, vitamins) may lead to the formulation of functional foods and nutraceuticals. With this aim, we have investigated the antioxidant properties of a dozen wild herbs that are among the compo- nents of "preboggion," a traditional dish of Liguria (northwestern Italy). These herbs were harvested from the seaside hills near Genoa. Among the wild herbs we have studied, at least six are characterized by radical scavenging activities, in terms of transfer of both $\mathrm{H}$ atoms and electrons, that are similar to or better than those of some foods that are well known for their antioxidant properties such as blueberries and red wine.

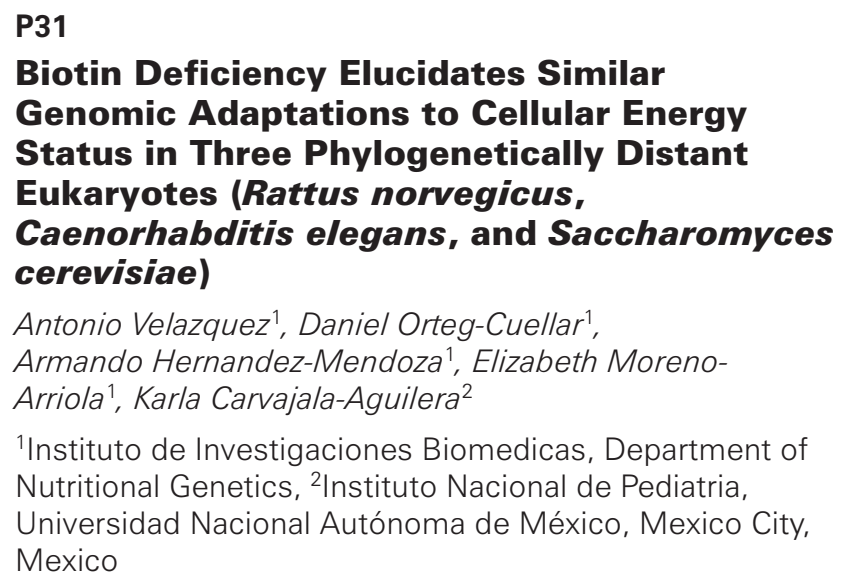

Objectives: Changes in genomic function drive flux modifications of [central carbon] metabolism by altering the amounts of enzymes and other proteins that catalyze the sequential steps of metabolic pathways. On the other hand, modifications of metabolism through other processes (allosterism, posttranscriptional modifications, epigenetic changes, etc.) can modulate genetic expression. However, the relationships between genetic expression and metabolism are complex and poorly understood.

Methods: We studied the effects of biotin deficiency versus sufficiency on the expression of the genes for enzymes of intermediary metabolism by means of DNA microarrays. The data were examined by means of Gene Set Enrichment Analysis (GSEA) in three diverse eukaryotes (Rattus norvegicus -"rat," Caenorhabditis elegans -"worm," and Saccharomyces cerevisiae -"yeast"). ATP, ADP, NAD, $\mathrm{NADH}$, pyruvate, lactate, ethanol, and oxygen consumption were measured by standard procedures.

Results: We found that biotin deprivation has a profound effect on genes of carbon metabolism. Although rat liver normally stores energy from dietary glucose largely as fat, its gene sets for glucose metabolism and for lipogenesis were significantly reduced in biotin deficiency in spite of its postprandial status, whereas the set for fatty acid oxidation was enriched. There were similar results in the deficient worm, together with reduction of those for TCA cycle and oxidative phosphorylation. Yeast uses glucose preferentially to other carbon sources whose utilization is repressed and has fermentative metabolism at the expense of downregulation of respiration when glucose is available, but in low biotin, paradoxically, transcripts for glucose oxidation were increased. All three deficient organisms consumed less oxygen and had low ATP levels. These results are likely due to reduced pyruvate carboxylase-driven TCA cycle anaplerosis. These changes were reversed when biotin was added to the deficient organisms. Also, in all three the transcripts for low-energy-sensing and -signaling proteins, such as AMPK/Snf1, TOR, and PGC1 $\alpha$, 
changed, as well as gene sets for rat adipocytokines, their corresponding JAK/STAT signaling pathway, and the insulin and the AMPKNAMPT-SIRT1 pathways.

Conclusions: These findings will likely be the starting point of a more comprehensive understanding of the evolutionary, increasingly more complex energy signaling networks and their effects on intermediary metabolism fluxes, as well as their derangements in diseases such as diabetes and cancer.

P32

\section{Hepatic Lipase -514C $>$ T Polymorphism and Its Association With High-Density Lipoprotein Cholesterol Level in Apparently Healthy Greek Adults: The ATTICA Study}

Nikos Yiannakouris ${ }^{1}$, Demosthenes B. Panagiotakos', Christos Pitsavos ${ }^{2}$, George Dedoussis', Christina Chrysohoou ${ }^{2}$, Eirini Louizou ${ }^{1}$, Eirini Theodoraki ${ }^{1}$ Marina Toutouza-Giotsa², Christodoulos Stefanadis ${ }^{2}$

${ }^{1}$ Harokopio University, Athens, Greece; ${ }^{2}$ University of Athens School of Medicine, Athens, Greece

Background and Objectives: The common $-514 \mathrm{C}>\mathrm{T}$ polymorphism of the hepatic lipase gene (LIPC) has been associated with low hepatic activity and high HDL-cholesterol concentrations. Moreover, a significant interaction between this polymorphism and dietary fat on HDL-cholesterol levels has been observed in different populations. We examined the association between the $L I P C-514 \mathrm{C}>\mathrm{T}$ polymorphism and plasma HDL-cholesterol concentrations in healthy Greek adults participating in the ATTICA study and evaluated whether this association was modified by dietary and clinical factors.

Methods: We studied demographic, lifestyle, clinical, biochemical, and genetic information from 548 men ( $41 \pm 11$ yrs) and 492 women ( $38 \pm 12 \mathrm{yrs})$ who had no evidence of cardiovascular or any other chronic disease. The association of the LIPC $-514 \mathrm{C}>\mathrm{T}$ polymorphism with plasma lipids was examined after taking into account the effect of several confounders. Adherence to the Mediterranean diet (MD) was evaluated through the MedDietScore.

Results: After adjustments for age, sex, smoking, body mass, and physical activity status, TT carriers ( $4.2 \%$ of all subjects) had higher HDL-cholesterol compared to CT (genotype frequency $=31.5 \%)$ and CC genotypes ( $52 \pm 13$ vs. $50 \pm 14$ vs. $48 \pm 13 \mathrm{mg} /$ $\mathrm{dL}, \mathrm{p}=0.037)$. The latter was evident in participants with normal glucose levels $(p=0.05)$, while significance was lost in those with glucose $>100 \mathrm{mg} / \mathrm{dL}(\mathrm{p}=0.66)$. Greater adherence to the MD (third vs. first quartile of MedDietScore) was associated with $22 \%$ higher HDLcholesterol levels ( $\mathrm{p}$ for trend $<0.001$ ); however, the effect of $L I P C$ -514 polymorphism on HDL levels was independent of the level of adherence to the MD ( $\mathrm{p}$ for interaction $=0.49)$. No significant associations were observed between $L I P C-514$ polymorphism and total cholesterol, LDL-cholesterol, and triglyceride levels (all p's $>0.47$ ). In addition, no significant gene $\mathrm{X}$ fat intake, gene $\mathrm{X}$ alcohol drinking, and gene $\mathrm{X}$ obesity status were observed (all p's $>0.10$ ).

Conclusion: Our results show that the $L I P C-514 \mathrm{C}>\mathrm{T}$ variant is associated with higher HDL-cholesterol levels in Greek adults, independent of dietary habits and obesity status.
P33

A Common Haplotype at the IL18 Locus Is Associated With Insulin Levels, Estimates of Insulin Resistance, and Postprandial Measures

\author{
Nikos Yiannakouris', Melissa C. Smart², \\ George Dedoussis', Marie-Lise Grisoni, ${ }^{3,4}$, Gie Ken Dror ${ }^{2}$, \\ Mary Yannakoulia ${ }^{1}$, Constantina Papoutsakis' \\ Eirini Louizou ${ }^{1}$, Labros Melistas ${ }^{1}$, Meropi D. Kontogianni ${ }^{1}$, \\ Jackie A. Cooper ${ }^{2}$, Steve E. Humphries ${ }^{2}$, \\ Philippa J. Talmud ${ }^{2}$, on behalf of the EARS// Consortium. \\ ${ }^{1}$ Harokopio University, Athens, Greece; ${ }^{2}$ Division of \\ Cardiovascular Genetics, British Heart Foundation \\ Laboratories, Department of Medicine, Royal Free and \\ UCL Medical School, London, UK; ${ }^{3}$ INSERM, UMR S 937, \\ F-75013, Paris, France; 'UPMC Univ Paris 06, UMR S 937, \\ F-75013, Paris, France
}

Objectives: Interleukin 18 (IL-18) has recently emerged as a metabolic factor and an adipogenic cytokine. Increased IL-18 expression has been observed in both type 2 diabetes and obesity, suggesting a possible role for IL-18 in glucose and energy homeostasis. In this study we investigated variation within the IL18 gene and its effect on markers of obesity and the metabolic syndrome.

Methods: Five IL-18 tagging single nucleotide polymorphisms (tSNPs) were selected (SNPs -9731G $>$ T, rs 1946519; -5848T $>$ C, rs2043055; +105A $>$ C, rs549908; +8855T $>$ A, rs360729; +11015, rs3882891) and genotyped in the Greek Obese Women Study (GrOW, $\mathrm{n}=349)$, the GENDAI study of Greek children $(\mathrm{n}=882)$, and the EARSII study of university students recruited in Europe $(n=822)$.

Results: Single SNP analysis showed no significant associations with BMI, measures of body fatness, or plasma glucose and lipid levels in any of the studies. However, significant associations were observed with the $-5848 \mathrm{~T}>\mathrm{C}$ variant and estimates of insulin resistance in the GrOW study. Subjects homozygous for the $-5848 \mathrm{C}$ variant exhibited higher insulin levels $(\mathrm{p}=0.05)$ and a higher HOMA-IR index $(p=0.04)$ in comparison to carriers for the common $\mathrm{T}$ allele. Six common haplotypes were observed in all of the studies. In the GrOW study, highly significant associations were observed at the haplotypic level with plasma insulin levels and estimates of insulin resistance. Hap6 (12222, frequency 2.6\%) was significantly associated with higher insulin levels $(\mathrm{p}<0.0001)$ and estimates of HOMA - -IR $(p<0.0001)$ and $\mathrm{HOMA}_{\beta \text {-cell }}(\mathrm{p}<0.0001)$ when compared with the most common haplotype, Hap1 (12111, frequency 33.2\%). None of the haplotypes were associated with any baseline measures in GENDAI or EARSII. However, an association with postprandial measures at the haplotypic level was observed in the EARSII study. Hap6 was associated with borderline significantly higher area under the curve TG after an oral fat tolerance test (global $p=0.06$ ).

Conclusion: We report for the first time the influence of genetic variation within IL-18 on insulin levels and measures of insulin resistance. The magnitudes of these effects are large and of a degree that could impact on individual risk of developing features of the metabolic syndrome. 
P34

\section{Combined Hereditary/Genetic and Epigenetic Aspects in Genetic Testing of Complex Diseases and Nutrigenetics}

S.D. Englert, A.G. Has/berger

Department of Nutritional Sciences, University of Vienna, Vienna, Austria

Background: Genetic testing and preventive health care based on the use of single nucleotide polymorphisms (SNPs) have been criticized because of methodological errors and low penetrance. The potential need to consider epigenetic aspects in the genetic analysis of complex diseases and nutritional disorders is being discussed. To evaluate epigenetic influences in genetic and nutrigenetic testing, interactions of SNPs and epigenetic DNA-methylation are analyzed.

Methods: The penetrance of selected SNPs with relevance to selected cancers is evaluated calculating odds ratio (OR) and p-value (p) in meta-analysis. In comparison, the relevance of DNA-methylation is assessed.

Results: Whereas penetrance of analyzed SNPs was usually found to be low, strong correlations of epigenetic DNA-methylation were found. For example, ORs of 1,5 ( $\mathrm{p} \approx 0.55$, after Bonferroni correction) were found for the common A148T variant of the tumor-suppressor gene p16 in colorectal cancer. In contrast, hypermethylation was found in about $30 \%$ of sporadic colorectal cancer. Hypermethylation of tumor-suppressor genes such as p16 is reversible by nutritional components such as Genistein in vitro.

Discussion: These findings underline the importance of integrating hereditary genetic aspects in concepts for genetic and nutrigenetic analyses in public health care.

\section{P35}

\section{Cellular and Molecular Effects of Dietary Fish Oil in Metabolic Syndrome Rat Models}

\author{
Rosamaría Oliart', Lyssia Castellanos², Ofelia Angulo', \\ Mauricio Rodríguez ${ }^{2}$, Silvia Berruezo ${ }^{1}$ \\ 'Instituto Tecnológico de Veracruz, Veracruz, México; \\ ${ }^{2}$ Instituto Nacional de Medicina Genómica, México DF, \\ México
}

Metabolic syndrome (MS), the major cause of morbidity and mortality in most of the world, is a genetically complex disorder heavily influenced by environmental and lifestyle factors whose molecular basis is largely unknown. It includes the clustering of abdominal obesity, insulin resistance, dyslipidemia, and elevated blood pressure and is associated with other comorbidities, including prothrombotic state, proinflammatory state, nonalcoholic fatty liver disease, and reproductive disorders.

There are a number of epidemiological studies supporting the dietary regulation of each of the metabolic risk factors of MS, and there is a great deal of interest in how dietary fat composition influences the development of MS. Among the dietary interventions, fish oil (rich in n-3 polyunsaturated fatty acids [n-3 PUFAs]) administration has proven to have several beneficial effects on MS components.
Objective: To evaluate the effects of dietary fish oil on the metabolic parameters and gene and protein expression levels in two MS rat models.

Methods: We used the spontaneously hypertensive rat (SHR) model and the sucrose-induced metabolic syndrome rat (SRM) model that was obtained by the administration of $30 \%$ sucrose in drinking water for 12 weeks. After the SRMs were established, the effects of the administration of dietary fish oil during 6 weeks were analyzed on the metabolic parameters of MS on the expression levels of the $C d 36$ gene and in protein expression patterns.

Results: In both models, after 6 weeks of fish oil administration, we found significant reductions in blood pressure, serum insulin, triacylglycerols, cholesterol, LDL, HDL, free fatty acids, and total lipids. We also found changes in membrane fatty acid composition in adipose tissue, liver, and brain, which could subsequently alter hormone signaling. Fish-oil-fed rats showed increased expression levels of the Cd36 gene, which codes for an integral membrane glycoprotein (Cd36) expressed on the surface of cells active in fatty acid metabolism and which participates in the uptake of long-chain fatty acids and oxidized LDL. It has been suggested that a Cd36 deficiency underlies insulin resistance, defective fatty acid metabolism, and hypertriglyceridemia. Additionally, we have found some proteins present after fish oil diet administration related to signal transduction pathways and cytoskeleton.

Conclusions: Our studies suggest that the modification of the membrane fatty acid composition, induction of $C d 36$ expression, and synthesis of proteins involved in signal transduction could be some of the cellular and molecular mechanisms elicited by fish oil PUFAs to ameliorate the metabolic syndrome components.

\section{P36 \\ Precisely Controlled Human Vitamin C Intake, Genomic Expression Patterns, and Immune Homeostasis}

\author{
Michael Graham Espey, Jin Wang, Maggie Cam, \\ Yahoui Wang, Sebastian Padayatty, Peter Eck, Mark Levine
}

Molecular and Clinical Nutrition Section, National Institute of Diabetes and Digestive and Kidney Diseases, National Institutes of Health, Bethesda, Md., USA

Objective: The potential for relationships between vitamin $\mathrm{C}$ (ascorbate) intake and gene expression in peripheral blood leukocytes was examined in human subjects.

Methods: Healthy volunteers $(n=11)$ were hospitalized for a 5-7 month period. Diet was restricted to menu selections that permitted individual preferences to be met, while providing all essential nutrients, with the exception of vitamin $\mathrm{C}(<5 \mathrm{mg} /$ day). Using a depletion/repletion design, blood samples then were sequentially obtained at steady state for doses of 30,60,100, and 2,500 $\mathrm{mg}$ of ascorbate daily over a period of weeks to months. Ascorbate in water was given in two divided doses in the fasted state to precisely control ascorbate intake. Blood samples were separated into plasma, lymphocyte, and monocyte fractions by apheresis. HPLC separation with electrochemical detection, human genomic microarray (Affymetrix U133 Plus 2.0), and real-time PCR validation techniques were used. 
Results and Conclusions: Ascorbate concentrations in plasma and leukocytes correlated with daily dose and uniformly followed saturation kinetics. Despite this, select leukocyte transcriptional networks changed (+ve, T-lymphocyte signaling; -ve, heat-shock pathways) in an ascorbate dose-dependent manner. Variation of genetic responses was also evident per individual, suggesting that the connectivity of specific nutrients to genomic expression patterns and immunity traits may be heterogeneous. Interindividual diversity in nutrient-transcriptome relationships may be evolutionarily based to facilitate phenotypic variation in immune responses to pathogens under different dietary conditions.

Significance: This unique data set relates precise changes in the intake of a specific nutrient (ascorbate) by the same human subject over time (months) to the person's individual gene expression patterns, which, to our knowledge, is the first of its kind for any component of our diet.
P37

\section{Plumbagin Promotes the Generation of Astrocytes From Embryonic Rat Neural Progenitors Via Activation of the Transcription Factor Stat3}

Yongquan Luo, Mohamed Mughal, Xin Ouyang, Haiyang Jiang, Tae-Gen Son, Mark P. Mattson

Laboratory of Neurosciences, Intramural Research Program, National Institute on Aging, National Institutes of Health, Baltimore, Md., USA

Plumbagin (PL, 5-hydroxy-2-methyl-1,4 naphthoquinone) is a naturally occurring low-molecular-weight lipophilic phytochemical derived from roots of plants of the Plumbago genus. PL has been reported to have several clinically relevant biological activities in non-neural cells, including antiatherosclerotic, anticoagulant, anticarcinogenic, antitumor, and bactericidal effects. In a recent screen of a panel of botanical pesticides, we identified PL as having neuroprotective activity. In the present study, we determined whether PL could modify the developmental fate of rat E14.5 embryonic neural progenitor cells (NPCs). PL exhibited no cytotoxicity when applied to cultured NPCs at concentrations below $1 \mu \mathrm{M}$. At a concentration of $0.1 \mu \mathrm{M}$, PL significantly enhanced proliferation of NPCs as indicated by a $17 \%$ increase in the percentage of cells incorporating BrdU incorporation. PL at a concentration of $0.1 \mathrm{pM}$ (but not $0.1 \mu \mathrm{M}$ ), stimulated astrogenesis as indicated by increased GFAP expression both at the mRNA and protein levels. The ability of PL to induce astrocyte production was further confirmed in anti-A2B5 enriched glial precursors. The phenotype of the cells that differentiated in response to PL, GFAP-positive cells with a flattened cell body and long processes, was characteristic of type 2 astrocytes. PL did not affect neurogenesis, as indicated in no change in levels of III tubulin, a mature neuron marker. PL $(0.1 \mathrm{pM})$ rapidly activated the transcription factor Stat 3 in NPCs, and a Stat3 inhibitor peptide prevented PL-induced astrocyte formation, showing that Stat 3 activation mediates PL-induced astrogenesis. These findings demonstrate the ability of a low-molecularweight, naturally occurring phytochemical to control the fate of neural progenitor cells by engaging a specific cellular signaling pathway. 


\section{Journal of \\ Nutrigenetics Nutrigenomics}

Aagaard, K. 196

Ahn, J. 194

Ahn, Y. 216

Algarve, T.D. 214

Alveranga-Lopez, J.C. 207

Andersson, N. 216

Angulo, O. 221

Antonelli, G.M. 211

Anzidei, P. 211

Arbo, I. 206

Arellano, A.V. 206

Arregui, M. 207

Ashibe, B. 215

Ashktorab, H. 214

Barberis, M. 214

Bastarrachea, R. 207

Beland, F.A. 200

Bergeron, J. 210

Berndt, S.I. 194

Berruezo, S. 221

Biunno, I. 214

Bouchard, C. 210

Bouchard, L. 210

Bowman, R. 213

Brattbakk, H.-R. 206

Brody, L.C. 199

Burdge, G. 195

Cabungcal, J.H. 201

Camargo, L.F.T. 209

Cam, M. 221

Campión, J. 206, 212

Carrasco, P. 207

Carvajala-Aguilera, K. 219

Castellanos, L. 221

Cattaneo, M. 214

Chang, C. 194

Chanock, S.J. 194

Chrysohoou, C. 220

Clark, R.M. 211

Cole, S. 207

Coltell, O. 207

Comuzzie, A. 207

Conus, P. 201

Cooper, J.A. 220

Corella, D. 207

Couture, P. 217

Croce, C.M. 211

Cross, A.J. 194

Cuenod, M. 201

Curi, R. 208, 209, 212, 217 , 219
Da Cruz, I.B.M. 209, 214

Davis, J.M. 195

de Cabo, R. 198

De Caterina, R. 190

Dedoussis, G. 220

De Marco, V. 219

De Miguel, C. 212

Deshaies, Y. 210

Després, J.P. 210

Do, K.Q. 201

Dolley, G. 210

Dotson, W.D. 204

Dowling, N.F. 204

Dror, G.K. 220

Duarte, M.F.F. 209

Duarte, T. 209

Eck, P. 221

Emmett, P.M. 195

Englert, S.D. 221

Espey, M.G. 221

Estruch, R. 207

Evelo, C. 202

Faergeman, O. 204

Farber, J.L. 211

Faucher, G. 210

Ferguson, L.R. 191, 205

Fernandez, M.-L. 211

Ferrucci, L.M. 194

Finzi, G. 214

Fong, L.Y. 211

Forey-Garcia, M. 207

Forsyth, C.B. 200

Fortes, C. 211

Frank, A. 201

Fujiwara, H. 208

Fu, Q. 196

Gabrielsson, B.G. 216

Garcia-Diaz, D.F. 206

Garenc, C. 217

Garneau, V. 210

Gillies, P.J. 205

Golding, J. 195

Gómez-Ruiz, A. 212

Gomiero, T. 219

Go, M.J. 216

Gorjäo, R. 212

Gottlieb, M.G. 209

Graubard, B.I. 194

Green, W. 214

Grisoni, M.-L. 220
Grove, K. 196

Guillen, M. 207

Gunter, M.J. 194

Gysin, R. 201

Hamid, R. 216

Hamilton, J.W. 198

Hampton, T. 198

Hankinson, S.E. 202

Haslberger, A.G. 221

Hatanaka, E. 208, 217

Hayes, R.B. 194

Hernandez-Mendoza, A. 219

Herron, K. 211

Hibbeln, J.R. 195

Higgins, P. 207

Hirabara, S.M. 209

Ho, E. 197

Hoshino, K. 212

Hould, F.-S. 210

Huang, W-Y. 194

Humphries, S.E. 220

Hunter, D.J. 190

Hunt, S.C. 193

Hu, R. 205

Innocenzi, L. 211

Jackson, A. 195

Jalali, S. 216

Jeffery, H. 213

Jehan, S. 216

Jiang, H. 222

Jiang, Y. 211

Johansen, B. 206

Joss-Moore, L. 196

Jung, I.E.C. 214

Kavanaugh, C.J. 203

Keshavarzian, A. 200

Ke, X. 196

Khaw, K.-T. 213

Kim, S.S. 216

Kohlmeier, M. 213

Kontogianni, M.D. 220

Kozul, C. 198

Krasnikov, B.F. 202

Kris-Etherton, P. 205

Kristal, B.S. 202

Kulseng, B. 206

Lamarche, B. 210

Lambertucci, R.H. 209
Lam, W.J. 205

Lane, R. 196

Langaas, M. 206

Lavoie, S. 201

Lebel, S. 210

Lee, E.L. 214

Levada-Pires, A.C. 217

Levine, M. 221

Lillycrop, K. 195

Lima, T.M. 217

Lindberg, F. 206

Liu, X.-P. 211

Lofgren, I.E. 211

Loh, Y.H. 213

Lotti, L.V. 214

Louizou, E. 220

Luben, R. 213

Luo, Y. 222

McKnight, R. 196

Maechler, P. 209

Magdalon, J. 208

Manica-Cattani, M.F. 214

Manku, M.S. 192

Marceau, P. 210

Mariani-Costantini, R. 214

Martínez, J.A. 193, 206, 212

Martin, R.L. 215

Marur, V. 202

Masi, L.N. 219

Mastroeni, S. 211

Matson, W.R. 202

Mattson, M.P. 222

Ma, X. 194

Mayne, S.T. 194

Melchi, F. 211

Melistas, L. 220

Milagro, F.I. 206, 212

Mitrou, P.N. 213

Moreno-Arriola, E. 219

Moresco, R.N. 209

Morgano, A. 214

Moschetta, A. 214

Motojima, K. 215

Mughal, M. 222

Munday, K. 205

Nachbar, R.T. 209

Nauman, N. 216

Ned, R.M. 204

Nouraie, M. 214

Oliart, R. 221

\section{KARGER}

$$
\text { (C) } 2010 \text { S. Karger AG, Basel }
$$

Fax +4161306 1234

E-Mail karger@karger.ch

www.karger.com 
Ordovas, J. 207, 211

Ortega, C. 207

Orteg-Cuellar, D. 219

Ouyang, X. 222

Padayatty, S. 221

Palmer, C.N.A. 192

Palsdottir, V. 216

Panagiotakos, D.B. 220

Paoletti, M.G. 219

Paolucci, U. 202

Papoutsakis, C. 220

Park, S.J. 216

Pasquini P. 211

Pearson, K.J. 198

Pérusse, L. 210

Pithon-Curi, T.C. 217

Pitsavos, C. 220

Pogribny, I.P. 200

Polari, A. 201

Pollin, T.I. 194

Pomp, D. 201

Portioli Silva, É.P. 219

Portoles, O. 207

Proffitt, M. 207

Raffaella, G. 211

Rahman, I. 197
Rainwater, D. 207

Ren, M.Q. 218

Ribeiro, E.E. 214

Rigo, A. 219

Rocha, J.B.T. 209

Rocha, M.I.U.M. 214

Rodrigues, H.G. 208

Rodríguez, M. 221

Rodwell, S. 213

Rossetti, S. 218

Rossetto, M. 219

Ross, S.A. 199

Rudkowska, I. 217

Russell, N. 202

Sacchetti, L. 219

Sacchi, N. 218

Salgado, T.M.L. 219

Sato, F.T. 208

Schetingera, M.R.C. 209

Sessa, F. 214

Shaheen, R. 216

Shami, S.A. 216

Shapira, N. 218

Sheldon, D. 202

Shi, H. 202

Shuldiner, A.R. 194

Shurubor, Y.I. 202
Silveira, A.F. 214

Silveira, L.R. 209

Sinha, R. 194

Smart, M.C. 220

Smith, G. D. 195

Sniatynski, M. 202

Snitker, S. 194

Somenzi, G. 218

Son, T.-G. 222

Sorli, J.V. 207

Spence, J.T. 203

Starlard-Davenport, A. 200

Steer, C.D. 195

Stefanadis, C. 220

Steullet, P. 201

Stone, A.C. 191

Strandvik, B. 216

Svensson, P-A. 198

Taccioli, C. 211

Tagliavacca, L. 218

Talmud, P.J. 220

Tang, Y. 200

Tchernof, A. 210

Teichmann, T. 201

Tejero, E. 207

Theodoraki, E. 220

Thompson, K.L. 199
Todokoro, M. 215

Toutouza-Giotsa, M. 220

Triggs, C.M. 205

Tryndyak, V. 200

Ulrich, C.M. 194

Vanzani, P. 219

Velame, F. 212

Velazquez, A. 219

Venanzetti, F. 211

Vinolo, M.A. 208

Vohl, M.-C. 210, 217

Voruganti, S. 207

Wang, J. 221

Wang, Y. 221

Wan, S.-G. 211

Waterland, R.A. 195

Wickman, A. 216

Wood, A. 213

Yannakoulia, M. 220

Yeager, M. 194

Yiannakouris, N. 220

Zeisel, S.H. 196 